\title{
Decidable first-order theories of one-step rewriting in trace monoids
}

\author{
Dietrich Kuske $^{1}$ and Markus Lohrey ${ }^{2}$ \\ 1 Technische Universität Dresden, \\ Institut für Algebra, \\ D-01062 Dresden, Germany \\ 2 Universität Stuttgart, \\ Institut für Formale Methoden der Informatik (FMI) \\ Universitätsstr. 38, D-70569 Stuttgart, Germany \\ lohreyeinformatik.uni-stuttgart.de
}

Keywords: trace monoids, rewriting, confluence, decidability

\begin{abstract}
We prove that the first-order theory of the one-step rewriting relation associated with a trace rewriting system is decidable but in general not elementary. This extends known results on semi-Thue systems but our proofs use new methods; these new methods yield the decidability of local properties expressed in first-order logic augmented by modulo-counting quantifiers. Using the main decidability result, we define several subclasses of trace rewriting systems for which the confluence problem is decidable.
\end{abstract}

\section{Introduction}

Various kinds of rewriting systems, like term rewriting systems [60] or semi-Thue systems [4] (also called string rewriting systems), received a lot of attention in mathematics and theoretical computer science and are still an active field of research. Historically, rewriting systems were introduced to solve word problems in certain structures [63]. By the work of Markov [43] and Post [54], this hope vanished as they presented semi-Thue systems with an undecidable word problem. Despite this result, there are plenty of rewriting systems with a decidable word problem, the most famous class being that of confluent and terminating systems [32]. In general, also confluence and termination are undecidable properties of a semi-Thue system, see [28] for termination and [1] for confluence. A large amount of research tries to identify sufficient conditions for confluence/termination of rewriting systems (cf. [60]), or to describe classes of rewriting systems where confluence/termination is decidable. For instance, the use of critical pairs [32] and Newman's Lemma [52] allows to decide confluence for terminating term rewriting systems.

Confluence and termination are typical properties of the one-step rewrite graph $(A, \rightarrow)$ : the set of nodes $A$ consists of the objects that are rewritten (e.g., words in case of a semi-Thue system or terms in case of a term rewriting system), and there exists an edge $a \rightarrow b$ if $a$ can be rewritten in one step into $b$. In order to express properties of the one-step rewrite graph $(A, \rightarrow)$, one may use logics, like for instance first-order logic (FO-logic) over the structure $(A, \rightarrow)$. But for the purpose of expressing properties like 


\begin{tabular}{c||c|c|c|} 
& FO-theory of $(A, \rightarrow)$ & FO-theory of $(A, \stackrel{*}{\rightarrow})$ & MSO-theory of $(A, \rightarrow)$ \\
\hline \hline term rewriting systems & undecidable [65] & undecidable & undecidable \\
\hline semi-Thue systems & decidable $[14,29]$ & undecidable & undecidable \\
\hline
\end{tabular}

Table 1.

\begin{tabular}{c||c|c|c|} 
& FO-theory of $(A, \rightarrow)$ & FO-theory of $(A, \stackrel{*}{\rightarrow})$ & MSO-theory of $(A, \rightarrow)$ \\
\hline \hline $\begin{array}{c}\text { ground term rewriting } \\
\text { systems }\end{array}$ & decidable & decidable [14] & undecidable [8] \\
\hline prefix semi-Thue systems & decidable & decidable & decidable [9] \\
\hline
\end{tabular}

Table 2.

confluence, FO-logic over $(A, \rightarrow)$ is too weak: confluence is a property of the reflexive transitive closure $\stackrel{*}{\rightarrow}$ of $\rightarrow$, but the former is in general not first-order definable in $(A, \rightarrow)$. Hence, in order to express confluence, one has to use FO-logic over $\left(A, \rightarrow^{*}\right)$ or, alternatively, monadic second-order logic (MSO-logic) over $(A, \rightarrow)$ [26] (which allows to define the reflexive transitive closure). Known (un)decidability results for the above mentioned logics in the case of term rewriting systems and semi-Thue systems, respectively, are collected in Table 1 . Hence, most theories are undecidable. Note that the undecidability of the FO-theory of $(A, \stackrel{*}{\rightarrow})$ (and hence the MSO-theory of $(A, \rightarrow)$ ) for term rewriting systems and semi-Thue systems, respectively, follows in fact from the undecidability of confluence for these systems. On the other hand, Treinen's result on the undecidability of the FO-theory of $(A, \rightarrow)$ for a specific term rewriting system uses a more subtle argument. The decidability result for the FO-theory of $(A, \rightarrow)$ in case of semi-Thue systems [14,29] uses the fact that the one-step rewrite relation of a semi-Thue system can be described by a two-tape automaton which moves its heads synchronously on both tapes.

To obtain decidable theories from one-step rewrite graphs, one may restrict the positions where rewrite steps may take place. If only prefixes of strings are allowed to be rewritten, one obtains prefix semi-Thue systems [6,9]. Prefix semi-Thue systems can be generalized from strings to terms, which results in ground term rewriting systems $[5,33,14]$. Table 2 collects (un)decidability results for these systems. The decidability results for ground term rewriting systems use automata theoretic methods similar to those mentioned in the previous paragraph [14], whereas the decidability of the MSO-theory of the one-step rewrite graph of a prefix semi-Thue system [9] is based on Rabin's tree theorem [57].

This paper investigates the first-order theory of the one-step rewrite graphs of trace rewriting systems, which generalize semi-Thue systems. Cartier and Foata [7] investigated the combinatorics of free partially commutative monoids that became later known as trace monoids. Mazurkiewicz [46] introduced them into computer science. 
They form a mathematically sound model for the concurrent behaviour of systems of high abstraction. Since trace monoids are a generalization of free monoids, it was tempting to extend the investigation of free monoids to free partially commutative monoids. This resulted, e.g., in the extensive consideration of recognizable and rational trace languages (cf. [18] for a collection of surveys on this field), trace equations $[20,44,17]$, and trace rewriting systems $[15,16,38-40]$.

Our main result states that for any finite trace rewriting system, the first-order theory of the associated one-step rewrite graph is decidable. Because of the non-local effects of trace rewriting (see Example 3.6), the automata-theoretic techniques from Dauchet and Tison [14] and Jacquemard [29] are not applicable here and we had to search for other ideas. The first is an application of Gaifman's locality theorem: the validity of a first-order sentence in a structure $\mathcal{S}$ depends on first-order properties of spheres around elements of $\mathcal{S}$. Since this theorem is effective, we were left with the question how to describe the set of traces that are centers of an $r$-sphere satisfying a given first-order formula. Our second idea was to describe the $r$-sphere around a trace in the dependence graph of this trace by a sentence of monadic second-order logic. Note that this logic does not speak about the infinite rewrite graph, but about a single finite dependence graph; therefore we refer to this technique as "internalization of the $r$-sphere". We show that this is indeed effectively possible. Hence, by a result of Thomas [62], this implies that the set of traces that are centers of an $r$-sphere satisfying a given first-order formula is recognizable. Taking these two ideas together, we obtain that the first-order theory of the one-step rewrite graph of any trace rewriting system is decidable (Corollary 3.9).

We actually show a more general result (cf. Theorem 3.8) since we do not only consider trace rewriting systems, but scattered rewrite rules. The idea is that of a parallel rewrite step where the intermediate factors of a trace have to satisfy some recognizable constraints and can be permuted as long as they are independent in the trace monoid. But the main reason for introducing these systems is that they occur naturally in the course of our proofs (see, e.g., Lemma 5.4).

The second step in our decidability proof, i.e., the interpretation of the $r$-sphere around a trace in the dependence graph of this trace does not only work for first-order logic, but also for its extension by modulo-counting quantifiers. Thus, we obtain that local properties of this modulo-counting logic are decidable (Theorem 5.14). The decidability of non-local properties is not answered in this paper, the missing tool is an analogue of Gaifman's locality theorem for this logic. Libkin [34-36] and Nurmonen [53] proved generalizations of a version of Gaifman's Theorem for this and other counting extensions of first-order logic, but we could not make these generalizations serve our purposes.

As mentioned above, the first step in our decidability proof is an application of Gaifman's Theorem. Gaifman's translation of a first-order sentence into a Boolean combination of local sentences is of very high complexity, i.e., our decision procedure is far from efficient. We also show that one cannot avoid this nonelementary complexity. To this aim, we "externalize" internal first-order properties of a trace into its 
$r$-sphere. This construction yields a trace rewriting system such that the corresponding one-step rewrite graph has a nonelementary first-order theory (Theorem 6.3). Thus, our use of Gaifman's translation does not lead to an unreasonable inefficiency. We actually show a slightly stronger result, namely that the set of valid local sentences for a fixed radius is not elementary. In other words, the complexity of the decision question is already present when restricting to local sentences.

This nonelementary lower bound is shown for a nontrivial independence alphabet and the proof does not carry over to semi-Thue systems. The decision procedure for semi-Thue systems from $[14,29]$ is not elementary since it uses automata complementation for any quantifier alternation in a formula. This does of course not imply that there is no better decision algorithm. We show a lower bound of doubly exponential nondeterministic time for this problem (Theorem 6.5). Again this lower bound holds for local sentences for a fixed radius.

In the last section, we return to the confluence problem for trace rewriting systems. For terminating rewriting systems, confluence and local confluence are equivalent. The problem with trace rewriting systems is that there can be infinitely many critical pairs which makes it impossible to check all of them in turn [16]. Even worse, by [51], it is undecidable whether a length-reducing trace rewriting system is confluent. On the other hand in $[16,40]$ several subclasses of terminating trace rewriting systems were identified for which confluence is decidable. Here we will present new classes with this property (Theorems 7.4, 7.5, and 7.7). In general, even local confluence is not a firstorder property of the one-step rewrite graph. The classes of trace rewriting systems we consider in this last section ensure that local confluence is effectively expressible by a sentence of first-order logic. This allows to apply our main result on the decidability of these first-order properties and therefore implies the decidability of confluence for these classes when restricted to terminating systems.

\section{General notations}

The cardinality of a set $A$ is denoted by $|A|$. The identity relation on $A$ is denoted by $\operatorname{Id}_{A}=\{(a, a) \mid a \in A\}$. Given a binary relation $\rightarrow \subseteq A \times A$ on $A$ and $n \geq 0$ we define the relation $\rightarrow^{n}$ inductively by $\rightarrow^{0}=\operatorname{Id}_{A}$ and $\rightarrow^{n}=\rightarrow^{n-1} \circ \rightarrow$. We define $\rightarrow \leq n=\bigcup_{0 \leq k \leq n} \rightarrow^{k}$. The reflexive and transitive closure of $\rightarrow$ is $\stackrel{*}{\rightarrow}=\bigcup_{k>0} \rightarrow^{k}$.

Given an alphabet $\Sigma, \Sigma^{*}$ is the set of all finite words over $\Sigma$, i.e., the free monoid generated by $\Sigma$. The empty word is denoted by $\epsilon$. For $s \in \Sigma^{*},|s|$ is the length of $s$. The alphabet $\operatorname{alph}(s)$ of a word $s$ is the set of all symbols that occur in $s$. For $\Gamma \subseteq \Sigma$ we denote by $\pi_{\Gamma}(s)$ the projection of the word $s$ onto the symbols in $\Gamma$.

The notion of a model is defined as usual in logic, see e.g. [27]. For a given model $\mathcal{A}=(A, F, R)$ where $F$ is a set of operations on $A$ and $R$ is a set of relations over $A$, the signature of $\mathcal{A}$ contains the equality symbol $=$, and for each $f \in F$ (resp. $r \in R$ ) it contains a function symbol of the same arity as $f$ (resp. a relation symbol of the same arity as $r$ ) that we denote without risk of confusion by $f$ (resp. $r$ ) as well. Terms, atomic formulae, and (general) formulae over the signature of $\mathcal{A}$ are defined as usual. 
For a first-order sentence $\varphi$ over the signature of $\mathcal{A}$, i.e., a first-order formula without free variables, we write $\mathcal{A}=\varphi$ if $\varphi$ evaluates to true in $\mathcal{A}$. Finally the first-order theory of $\mathcal{A}$ denotes the set of all first-order sentences $\varphi$ over the signature of $\mathcal{A}$ with $\mathcal{A} \models \varphi$.

\section{Rewriting in trace monoids}

\subsection{Trace monoids and recognizable trace languages}

In the following we introduce some notions from trace theory, see [18] for more details. An independence relation on an alphabet $\Sigma$ is an irreflexive and symmetric relation $I \subseteq \Sigma \times \Sigma$, the complementary relation $D=(\Sigma \times \Sigma) \backslash I$ is called a dependence relation. The pair $(\Sigma, I)$ (resp. $(\Sigma, D))$ is called an independence alphabet (resp. a dependence alphabet). Given an independence alphabet $(\Sigma, I)$, we define the trace monoid $\mathbb{M}(\Sigma, I)$ as the quotient monoid $\Sigma^{*} / \equiv_{I}$ where $\equiv_{I}$ denotes the least congruence relation on $\Sigma^{*}$ that contains all pairs of the form $(a b, b a)$ for $(a, b) \in I$. An element of $\mathbb{M}(\Sigma, I)$, i.e., an equivalence class of words, is called a trace. The trace that contains the word $s$ is denoted by $[s]_{I}$. The neutral element of $\mathbb{M}(\Sigma, I)$ is the empty trace $[\epsilon]_{I}$ which will be denoted by 1 . Concatenation of traces is defined by $[s]_{I}[t]_{I}=[s t]_{I}$. If we want to speak explicitly of the concatenation as a binary operation over $\mathbb{M}$ then we denote it as $\circ$. A subset of $\mathbb{M}(\Sigma, I)$ is also called a trace language. The concatenation of two trace languages $L_{1}, L_{2} \subseteq \mathbb{M}(\Sigma, I)$ is the trace language $L_{1} L_{2}=\left\{[s]_{I}[t]_{I} \mid[s]_{I} \in L_{1},[t]_{I} \in L_{2}\right\}$.

For the rest of the paper let us fix an independence alphabet $(\Sigma, I)$ with $\Sigma$ finite and let $\mathbb{M}=\mathbb{M}(\Sigma, I)$ and $D=(\Sigma \times \Sigma) \backslash I$. Since for all words $s, t \in \Sigma^{*}, s \equiv_{I} t$ implies $|s|=|t|$ and $\operatorname{alph}(s)=\operatorname{alph}(t)$, we can define $\left|[s]_{I}\right|=|s|$ and $\operatorname{alph}\left([s]_{I}\right)=\operatorname{alph}(s)$. For a trace language $L \subseteq \mathbb{M}$ we define $\operatorname{alph}(L)=\bigcup_{u \in L} \operatorname{alph}(u)$. The independence relation $I$ can be lifted to $\mathbb{M}$ by $(u, v) \in I$ if $\operatorname{alph}(u) \times \operatorname{alph}(v) \subseteq I$. If $I=\emptyset$ then $\mathbb{M}$ is isomorphic to the free monoid $\Sigma^{*}$. On the other hand if $D=\operatorname{Id}_{\Sigma}$, then $\mathbb{M}$ is isomorphic to the free commutative monoid $\mathbb{N}^{|\Sigma|}$.

Traces can be uniquely represented as dependence graphs: the dependence graph $D_{t}=(V, E, \lambda)$ of a trace $t=\left[a_{1} \cdots a_{n}\right]_{I} \in \mathbb{M}$, where $a_{1}, \ldots, a_{n} \in \Sigma$, is defined as follows:

- $V=\{1, \ldots, n\}$ is the set of nodes.

- $E=\left\{(i, j) \mid i<j \wedge\left(a_{i}, a_{j}\right) \in D\right\}$ is the edge relation.

$-\lambda(i)=a_{i}$ for $1 \leq i \leq n$ defines the node labeling function $\lambda: V \rightarrow \Sigma$.

Then $D_{t}$ is well defined in the sense that if we choose another word that represents the trace $t$ then we obtain an isomorphic graph. Since $D_{t}$ is acyclic, the transitive reflexive closure $E^{*}$ of $E$ is a partial order. A subset $U \subseteq V$ such that

$$
\forall i, j \in U \forall k \in V: \text { if }(i, k),(k, j) \in E^{*} \text { then } k \in U
$$

is called a factor of $D_{t}$. Factors of a dependence graph are also called convex sets. Note that the intersection of two convex sets is again convex. For a convex $U \subseteq V$ 
there exists a unique $u \in \mathbb{M}$ such that the restriction $D_{t} \uparrow_{U}$ of $D_{t}$ to $U$ is isomorphic to $D_{u}$ and $t$ can be factorized as $t=t_{1} u t_{2}$. Vice versa if $t$ can be factorized as $t=t_{1} u t_{2}$ then there exists a convex $U \subseteq V$ such that $D_{t} \uparrow_{U}$ is isomorphic to $D_{u}$. More generally given a trace $t=t_{1} t_{2} \cdots t_{m}$, where $t_{i} \in \mathbb{M}$, the set of nodes $V$ of the dependence graph $D_{t}=(V, E, \lambda)$ can be partitioned into convex sets $V_{1}, \ldots, V_{m} \subseteq V$ such that $D_{t_{i}}$ is isomorphic to $D_{t} \uparrow_{V_{i}}$ and for all $1 \leq i<j \leq m$ there do not exist $p \in V_{i}$, $q \in V_{j}$ with $(q, p) \in E^{*}$. Vice versa given a partition of $V$ with these properties, the trace $t$ can be factorized as $t=t_{1} t_{2} \cdots t_{m}$. Hence we call a sequence of convex sets $V_{1}, \ldots, V_{m} \subseteq V$ with the properties above a factorization of $D_{t}$.

Lemma 3.1. Let $U_{1}, \ldots, U_{m}$ and $V_{1}, \ldots, V_{n}$ be two factorizations of the dependence graph $D_{t}$. If $1 \leq i<k \leq m$ and $n \geq j>\ell \geq 1$ then $\lambda\left(U_{i} \cap V_{j}\right) \times \lambda\left(U_{k} \cap V_{\ell}\right) \subseteq I$.

Proof. Let $1 \leq i<k \leq m, n \geq j>\ell \geq 1, p \in U_{i} \cap V_{j}$, and $q \in U_{k} \cap V_{\ell}$. Suppose that $(\lambda(p), \lambda(q)) \in D$. Hence, by definition of $D_{t}$, either $(p, q) \in E$ or $(q, p) \in E$. However, by the definition of factorization, $i<k$ implies that $(q, p) \notin E^{*}$, and $\ell<j$ implies that $(p, q) \notin E^{*}$.

The following lemma is known as Levi's Lemma for traces, see e.g. [13]. It can be easily deduced from Lemma 3.1.

Lemma 3.2. For $u_{1}, \ldots, u_{m}, v_{1}, \ldots, v_{n} \in \mathbb{M}, u_{1} u_{2} \cdots u_{m}=v_{1} v_{2} \cdots v_{n}$ if and only if there exist $w_{i, j} \in \mathbb{M}(1 \leq i \leq m, 1 \leq j \leq n)$ such that

- $u_{i}=w_{i, 1} w_{i, 2} \cdots w_{i, n}$ for every $1 \leq i \leq m$,

- $v_{j}=w_{1, j} w_{2, j} \cdots w_{m, j}$ for every $1 \leq j \leq n$, and

- $\left(w_{i, j}, w_{k, \ell}\right) \in I$ if $1 \leq i<k \leq m$ and $n \geq j>\ell \geq 1$.

A clique covering of the dependence alphabet $(\Sigma, D)$ is a sequence $\left(\Gamma_{1}, \ldots, \Gamma_{n}\right)$ of subsets of $\Sigma$ such that $\Sigma=\bigcup_{i=1}^{n} \Gamma_{i}$ and $D=\bigcup_{i=1}^{n} \Gamma_{i} \times \Gamma_{i}$. For a fixed clique covering $\left(\Gamma_{1}, \ldots, \Gamma_{n}\right)$ of $(\Sigma, D)$ we denote with $\pi_{i}: \mathbb{M} \rightarrow \Gamma_{i}^{*}$ the canonical lifting of the projection $\pi_{\Gamma_{i}}: \Sigma^{*} \rightarrow \Gamma_{i}^{*}$ to $\mathbb{M}$. The following lemma is well known, see e.g. [12].

Lemma 3.3. Let $\left(\Gamma_{1}, \ldots, \Gamma_{n}\right)$ be a clique covering of the dependence alphabet $(\Sigma, D)$. Then for $s, t \in \Sigma^{*}$ we have $s \equiv_{I} t$ if and only if $\pi_{i}(s)=\pi_{i}(t)$ for all $1 \leq i \leq n$.

A tuple $\left(s_{1}, \ldots, s_{n}\right) \in \prod_{i=1}^{n} \Gamma_{i}^{*}$ is reconstructible if there exists a trace $u \in \mathbb{M}$ such that $\pi_{i}(u)=s_{i}$ for all $i \in\{1, \ldots, n\}$ [12]. The following statement is from [20, Prop. 1.6].

Lemma 3.4. Let $u \in \mathbb{M}$, and let $\left(\Gamma_{1}, \ldots, \Gamma_{n}\right)$ be a clique covering of the dependence alphabet $(\Sigma, D)$. For all $i \in\{1, \ldots, n\}$ let $\pi_{i}(u)=s_{i} t_{i}$ be a factorization of $\pi_{i}(u)$. Then $\left(s_{1}, \ldots, s_{n}\right)$ and $\left(t_{1}, \ldots, t_{n}\right)$ are reconstructible if and only if $\pi_{i}\left(s_{j}\right)=\pi_{j}\left(s_{i}\right)$ for all $i, j \in\{1, \ldots, n\}$.

A set $L \subseteq \mathbb{M}$ is called recognizable if there exist a (monoid) morphism $h: \mathbb{M} \rightarrow Q$ from $\mathbb{M}$ into a finite monoid $Q$ and a subset $F \subseteq Q$ such that $L=h^{-1}(F)$. The set of all recognizable subsets of $\mathbb{M}$ is denoted by $\operatorname{REC}(\mathbb{M})$. The set $\operatorname{REC}(\mathbb{M})$ is effectively closed under Boolean operations and concatenation of trace languages. ${ }^{1}$ Furthermore,

\footnotetext{
${ }^{1}$ In these effectiveness statements, a recognizable language is given as a triple $(Q, F, h)$.
} 
if $\Gamma \subseteq \Sigma$ then $\{t \in \mathbb{M} \mid \operatorname{alph}(t) \subseteq \Gamma\}$ is recognizable. If it is clear that we want to denote a subset of $\mathbb{M}$ then we denote the latter set by $\Gamma^{*}$. Finally, emptiness and finiteness are decidable for recognizable trace languages, and if $L \in \mathrm{REC}(\mathbb{M})$ is finite then we can effectively calculate a list of all elements of $L$.

\subsection{Scattered trace rewriting}

Let us fix a countably infinite set $\Omega$ of (first-order) variables ranging over $\mathbb{M}$ for the rest of this paper. In order to make notations more succinct, we associate with every first-order variable $x \in \Omega$ a recognizable trace language $L(x)$ and require that $x$ ranges over $L(x)$, only. We assume that for every $L \in \operatorname{REC}(\mathbb{M})$ there is a countably infinite supply of variables $x \in \Omega$ with $L(x)=L$. Since $\operatorname{REC}(\mathbb{M})$ is countable, a countable set $\Omega$ of variables suffices for this. The mapping $x \mapsto L(x)$ will be fixed for the rest of this paper. For $x \in \Omega$, let $\operatorname{alph}(x)=\operatorname{alph}(L(x))$. On the set $\Omega$ we define an independence relation $J$ by

$$
J=\{(x, y) \mid \operatorname{alph}(x) \times \operatorname{alph}(y) \subseteq I\} \backslash \operatorname{Id}_{\Omega} .
$$

Let $x_{1}, \ldots, x_{m}$ be pairwise different variables from $\Omega$. A pattern $S$ over $\mathbb{M}$ and the variables $x_{1}, \ldots, x_{m}$ is a sequence $y_{1} t_{1} y_{2} t_{2} \cdots y_{m}$ where $t_{j} \in \mathbb{M}(1 \leq i \leq m-1)$ and the function $x_{i} \mapsto y_{i}(1 \leq i \leq m)$ is a permutation of $\left\{x_{1}, \ldots, x_{m}\right\}$. A pattern $S$ over the variables $x_{1}, \ldots, x_{m}$ is also denoted by $S\left(x_{1}, \ldots, x_{m}\right)$. Note that in a pattern a variable occurs precisely once, but the variables may occur in an arbitrary order. If the variable $x_{i}$ evaluates to $u_{i} \in \mathbb{M}, 1 \leq i \leq m$, then the trace $S\left(u_{1}, \ldots, u_{m}\right) \in \mathbb{M}$ is defined in the obvious way. By type $(S)$ we denote the projection of the pattern $S=y_{1} t_{1} y_{2} t_{2} \cdots y_{m}$ onto its variables, i.e., $\operatorname{type}(S)=y_{1} y_{2} \cdots y_{m}=\pi_{\Omega}(S)$ if we view $S$ as a word over the infinite alphabet $\mathbb{M} \cup \Omega$. A scattered rewrite rule over $\mathbb{M}$ and the variables $x_{1}, \ldots, x_{m}$ is a pair $\left(S\left(x_{1}, \ldots, x_{m}\right), T\left(x_{1}, \ldots, x_{m}\right)\right)$ where $S$ and $T$ are patterns over $\mathbb{M}$ such that type $(S) \equiv_{J}$ type $(T)$. The set of all scattered rewrite rules over $\mathbb{M}$ (and an arbitrary sequence of variables) is denoted by $\mathbb{S}$. A scattered rewriting system over $\mathbb{M}$ is a finite subset of $\mathbb{S}$. For a scattered rewrite rule $\rho=\left(S\left(x_{1}, \ldots, x_{m}\right), T\left(x_{1}, \ldots, x_{m}\right)\right)$ and $s, t \in \mathbb{M}$ we write $s \rightarrow_{\rho} t$ if there exist $u_{1} \in L\left(x_{1}\right), \ldots, u_{m} \in L\left(x_{m}\right)$ such that $s=S\left(u_{1}, \ldots, u_{m}\right)$ and $t=T\left(u_{1}, \ldots, u_{m}\right)$. For a scattered rewriting system $\mathcal{R}$ we write $s \rightarrow_{\mathcal{R}} t$ if $s \rightarrow_{\rho} t$ for some $\rho \in \mathcal{R}$. Note that in a scattered rewriting rule $\left(S\left(x_{1}, \ldots, x_{m}\right), T\left(x_{1}, \ldots, x_{m}\right)\right)$ we may rename the variables $x_{1}, \ldots, x_{m}$ into $y_{1}, \ldots, y_{m}$ as long as $L\left(x_{i}\right)=L\left(y_{i}\right)$ for every $i \in\{1, \ldots, m\}$.

Example 3.5. Let $(\Sigma, I)$ be the following independence alphabet:

$$
b-d-a-c
$$

The corresponding dependence alphabet is

$$
a-b-c-d \text {. }
$$


Let $x, y, z \in \Omega$ such that $L(x)=\{b, c\}^{*}, L(y)=\{a, b\}^{*}$, and $z \in\{d\}^{*}$. Note that $\operatorname{alph}(y) \times \operatorname{alph}(z) \subseteq I$. Hence, $\rho=(x a c y b d z, x b d z a c y)$ is a scattered rewrite rule. By substituting $b c$ for $x, a b b$ for $y$, and $d$ for $z$, respectively, we obtain

$$
b c a c a b b b d d \rightarrow_{\rho} b c b d d a c a b b \text {. }
$$

An important special case of scattered rewriting systems are trace rewriting systems $[15,16]$. A trace rewriting system over $\mathbb{M}$ may be defined as a scattered rewriting system $\mathcal{R}$ over $\mathbb{M}$ where all rules $\rho \in \mathcal{R}$ have the form $(x \ell y, x r y)$ for $\ell, r \in \mathbb{M}$ and $x, y \in \Omega$ with $L(x)=L(y)=\mathbb{M}$. If $I=\emptyset$, i.e., $\mathbb{M} \simeq \Sigma^{*}$, then a trace rewriting system over $\mathbb{M}$ is also called a semi-Thue system over $\Sigma^{*}$, see [30,4] for more details. On the other hand if $I=(\Sigma \times \Sigma) \backslash \operatorname{Id}_{\Sigma}$, i.e., $\mathbb{M} \simeq \mathbb{N}^{|\Sigma|}$, then a trace rewriting system over $\mathbb{M}$ is also called a vector replacement system over $\mathbb{N}^{|\Sigma|}$. A rule $(x \ell y, x r y)$ of a trace rewriting system will be briefly denoted by $(\ell, r)$.

The application of a trace rewriting system (and hence also a scattered rewriting system) to a trace may have non-local effects. This is demonstrated in the following example.

Example 3.6. Let $\Sigma=\{a, b, c\}$ and $I=\{(a, c),(c, a)\}$. Let $\mathcal{R}=\{(b, 1),(1, b)\}$, which is considered as a trace rewriting system over $\mathbb{M}(\Sigma, I)$. Then we have

$$
a^{m} b c^{n} \rightarrow_{\mathcal{R}} a^{m} c^{n}=c^{n} a^{m} \rightarrow_{\mathcal{R}} c^{n} b a^{m}
$$

for all $m, n \in \mathbb{N}$. Since $b$ is dependent from $a$ and $c$, no commutations can take place in the traces $a^{m} b c^{n}$ and $c^{n} b a^{m}$, i.e., these two traces behave like words. But the difference between the words $a^{m} b c^{n}$ and $c^{n} b a^{m}$ cannot be detected in a local way. More formally, the binary relation $\left\{\left(a^{m} b c^{n}, c^{n} b a^{m}\right) \mid m, n \geq 0\right\}$ is not a synchronized rational relation in the sense of [24].

Next we will introduce different models, whose domain will be the set $\mathbb{M}$ of all traces. The most general model we will work with is the model

$$
\mathcal{M}=\left(\mathbb{M}, \circ,(a)_{a \in \Sigma \cup\{1\}},(K)_{K \in \operatorname{REC}(\mathbb{M})},\left(\rightarrow_{\rho}\right)_{\rho \in \mathbb{S}}\right) .
$$

The operations of this model are the concatenation of traces $\circ$ plus the constants $a \in$ $\Sigma \cup\{1\}$. Furthermore each recognizable language $K \in \operatorname{REC}(\mathbb{M})$ is put into $\mathcal{M}$ as a unary predicate. Instead of $K(x)$ we write $x \in K$. Finally $\mathcal{M}$ contains all binary relations $\rightarrow_{\rho} \subseteq \mathbb{M} \times \mathbb{M}$ for $\rho \in \mathbb{S}$.

If $D=\mathrm{Id}_{\Sigma}$ then the monoid $\mathbb{M}$ is isomorphic to the free commutative monoid $\mathbb{N}^{|\Sigma|}$, whose first-order theory is decidable [55]. Since any recognizable set in $\mathbb{N}^{|\Sigma|}$ is semilinear and hence first-order definable in $\mathbb{N}^{|\Sigma|}$, it follows that the unary predicates $(K)_{K \in \operatorname{REC}(\mathbb{M})}$ and the binary predicates $\left(\rightarrow_{\rho}\right)_{\rho \in \mathbb{S}}$ are all first-order definable in $\mathbb{N}^{|\Sigma|}$. Thus, in case $D=\operatorname{Id}_{\Sigma}$ the first-order theory of $\mathcal{M}$ is decidable. Now suppose there are $a, b \in \Sigma$ with $(a, b) \in D$ and $a \neq b$. Then the structure $\left(\{a, b\}^{*}, \circ\right)$ can be interpreted by a first-order formula in $\mathcal{M}$. Since the first-order theory of $\left(\{a, b\}^{*}, \circ\right)$ is undecidable $[56,42,21]$, we get 
Proposition 3.7. The first-order theory of $\mathcal{M}$ is decidable if and only if $D=I d_{\Sigma}$.

Note that the undecidability arises from the monoid operation. Therefore, we will concentrate on reducts of $\mathcal{M}$ where the signature is restricted to the other relations. For a scattered rewriting system $\mathcal{R} \subseteq \mathbb{S}$ we define:

$$
\begin{aligned}
& \mathcal{M}^{\text {red }}=\left(\mathbb{M},(K)_{K \in \operatorname{REC}(\mathbb{M})},\left(\rightarrow_{\rho}\right)_{\rho \in \mathbb{S}}\right) \\
& \mathcal{M}_{\mathcal{R}}^{\text {red }}=\left(\mathbb{M},(K)_{K \in \operatorname{REC}(\mathbb{M})},\left(\rightarrow_{\rho}\right)_{\rho \in \mathcal{R}}\right)
\end{aligned}
$$

Note that the constants $a \in \Sigma \cup\{1\}$ can be defined in any of these models since the set $\{a\}$ is recognizable. Since they do not play any prominent role in our considerations of $\mathcal{M}_{\mathcal{R}}^{\text {red }}$ (as opposed to those of $\mathcal{M}$ ), we omit them in the signature.

For a scattered rewriting system $\mathcal{R}$, one may consider the reduct $\left(\mathbb{M},\left(\rightarrow_{\rho}\right)_{\rho \in \mathcal{R}}\right)$ of $\mathcal{M}_{\mathcal{R}}^{\text {red }}$ (the one-step rewrite graph of $\mathcal{R}$ ) as an edge-labeled directed graph: the relation $\rightarrow_{\rho}$ contains all edges that are labeled with the rule $\rho$. Let us end this section with a brief comparison of these graphs with other well-known classes of (infinite) graphs.

A graph is called context-free if it is the transition graph of a pushdown automaton [50]. In [9], Caucal gave an alternative characterization using prefix semi-Thue systems. Let us define a prefix rewriting system over $\mathbb{M}$ as a scattered rewriting system $\mathcal{R}$ where all rules in $\mathcal{R}$ have the form $(x \ell y, x r y)$ with $L(x)=\{1\}$ and $L(y)=\mathbb{M}$ (again we abbreviate this rule by $(\ell, r)$ ). Thus reductions may only occur in the prefix of a trace. In case $I=\emptyset$, prefix rewriting systems are called prefix semi-Thue systems. Caucal has shown that an edge-labeled directed graph is context-free if and only if it is isomorphic to a graph $\left(\Sigma^{*},\left(\rightarrow_{\rho}\right)_{\rho \in \mathcal{R}}\right)$ for a prefix semi-Thue system $\mathcal{R}$. It follows that the class of context-free graphs is contained in the class of one-step rewrite graphs of scattered rewriting systems. Moreover, containment is strict, because the MSO-theory of a context-free graph is decidable [50], which in general is not the case for one-step rewrite graphs of semi-Thue systems, see Table 1.

By considering rewriting on trees (terms) instead of words, prefix semi-Thue systems generalize to ground tree rewriting systems. The one-step rewrite graphs of the latter class of systems were studied in $[14,8,37]$. The decidability results of Tables 1 and 2 imply that these graphs do not cover the one-step rewrite graphs of semiThue systems, and hence not those of scattered rewriting systems. Another proper generalization of the class of context-free graphs is the class of prefix-recognizable graphs [10], see [2] for a variety of different characterizations. The class of prefixrecognizable graphs is incomparable to the class of one-step rewrite graphs of scattered rewriting systems (as well as to the class of one-step rewrite graphs of ground tree rewriting systems): the MSO-theory of a prefix-recognizable graph is still decidable; on the other hand, prefix-recognizable graphs may contain nodes of infinite degree which is not the case for one-step rewrite graphs of scattered rewriting systems.

Prefix-recognizable graphs can be generalized to automatic graphs [31]. Roughly speaking, a graph is automatic if the nodes can be represented by words from a regular word language, and the edge relations can be recognized by two-tape automata 
with synchronously moving heads. Finally, let us mention that automatic graphs were generalized to rational graphs $[48,49]$. They are also defined via two-tape automata which, in contrast to automatic graphs, are not required to move synchronously. For $I=\emptyset$, the one-step rewrite graph of a scattered rewriting system is automatic. In the general case, we do not even know whether the one-step rewrite graph of a scattered rewriting system is at least rational.

\subsection{The main result}

The main result of this paper states that the first-order theory of any structure $\mathcal{M}^{\text {red }}$ is decidable. Since our decision procedure is uniform in the underlying alphabet, we obtain

Theorem 3.8. There is an algorithm that, on input of an independence alphabet $(\Sigma, I)$ and a first-order sentence $\varphi$ over the signature of the structure

$$
\mathcal{M}^{\mathrm{red}}=\left(\mathbb{M},(K)_{K \in \operatorname{REC}(\mathbb{M})},\left(\rightarrow_{\rho}\right)_{\rho \in \mathbb{S}}\right),
$$

decides whether $\mathcal{M}^{\text {red }} \models \varphi$.

The remainder of this section discusses this result and puts it into context. The following sections up to Section 5.4 prepare the proof of this theorem which can be found in Section 5.4.

Since any trace rewriting system is a scattered rewriting system, we obtain the following corollary of Theorem 3.8:

Corollary 3.9. There is an algorithm that, on input of an independence alphabet $(\Sigma, I)$, a trace rewriting system $\mathcal{R}$ over $\mathbb{M}=\mathbb{M}(\Sigma, I)$, and a first-order sentence $\varphi$ over the signature of the model $\left(\mathbb{M}, \rightarrow_{\mathcal{R}}\right)$, decides whether $\left(\mathbb{M}, \rightarrow_{\mathcal{R}}\right) \models \varphi$.

This corollary will be used later in Section 7 where we consider confluence problems for trace rewriting systems. As already mentioned in the introduction, Corollary 3.9 was already shown for semi-Thue systems by using the fact that for a semi-Thue system $\mathcal{R}$ the relation $\rightarrow_{\mathcal{R}}$ is a synchronized rational relation [24], i.e., can be recognized by a two-tape automaton where both heads move synchronously [14,29]. It is not hard to see that this method can be generalized to the case that $\mathbb{M}$ is a direct product of free monoids (this covers in particular the case of vector replacement systems), but there seems to be no way to generalize this approach to arbitrary trace monoids (see also Example 3.6). Hence in our proof of Theorem 3.8 we will follow a completely different and new strategy.

Next we show that Corollary 3.9 is a proper generalization of the semi-Thue case. More precisely, we construct a trace rewriting system $\mathcal{R}$ such that the graph $\left(\mathbb{M}, \rightarrow_{\mathcal{R}}\right)$ is not isomorphic to the graph of any semi-Thue system. For this we have to introduce some notions concerning confluence. Let $\mathcal{R}$ be a trace rewriting system. We say that $\mathcal{R}$ is locally confluent if for all $u, v_{1}, v_{2} \in \mathbb{M}$ with $u \rightarrow_{\mathcal{R}} v_{1}$ and $u \rightarrow_{\mathcal{R}} v_{2}$ there exists 
$w \in \mathbb{M}$ with $v_{1} \stackrel{*}{\rightarrow}_{\mathcal{R}} w$ and $v_{2} \stackrel{*}{\rightarrow}_{\mathcal{R}} w$. We say that $\mathcal{R}$ is $\alpha$-confluent, where $\alpha \in \mathbb{N}$, if for all $u, v_{1}, v_{2} \in \mathbb{M}$ with $u \rightarrow_{\mathcal{R}} v_{1}$ and $u \rightarrow_{\mathcal{R}} v_{2}$ there exists $w \in \mathbb{M}$ with $v_{1} \rightarrow{ }_{\mathcal{R}}^{\leq \alpha} w$ and $v_{2} \rightarrow \stackrel{<\alpha}{\mathcal{R}} w$.

Lemma 3.10. If a semi-Thue system is locally confluent then it is $\alpha$-confluent for some $\alpha \in \mathbb{N}$.

Proof. For every semi-Thue system $\mathcal{S}$ over $\Sigma^{*}$ there exist finitely many critical pairs $\left(u_{1}, v_{1}\right), \ldots,\left(u_{n}, v_{n}\right) \in \Sigma^{*} \times \Sigma^{*}$ which result from overlapping left-hand sides. Since $\mathcal{S}$ is locally confluent, for every critical pair $\left(u_{i}, v_{i}\right)$ there exists $w_{i} \in \Sigma^{*}$ such that $u_{i} \stackrel{*}{\rightarrow}_{\mathcal{S}} w_{i}$ and $v_{i} \stackrel{*}{\rightarrow}_{\mathcal{S}} w_{i}$, see e.g. [4]. Then there exists $\alpha \in \mathbb{N}$ such that $u_{i} \rightarrow_{\mathcal{S}} \leq \alpha$ $w_{i}$ and $v_{i} \rightarrow{ }_{\mathcal{S}}^{\leq \alpha} w_{i}$. From the definition of critical pairs and $\alpha$-confluence, it follows immediately that $\mathcal{S}$ is $\alpha$-confluent.

Theorem 3.11. Let $\Sigma=\{a, b, c\}, I=\{(a, c),(c, a)\}$, and let $\mathcal{R}$ be the trace rewriting system $\mathcal{R}=\{(b a, 1),(a b, 1),(c, 1)\}$ over $\mathbb{M}(\Sigma, I)$. Then there is no semi-Thue system $\mathcal{S}$ over some alphabet $\Gamma$ such that the graph $\left(\mathbb{M}(\Sigma, I), \rightarrow_{\mathcal{R}}\right)$ is isomorphic to the graph $\left(\Gamma^{*}, \rightarrow_{\mathcal{S}}\right)$.

Proof. In [40, Example 2.2] the second author has shown that $\mathcal{R}$ is locally confluent. We claim that there is no $\alpha \in \mathbb{N}$ such that $\mathcal{R}$ is $\alpha$-confluent; this proves the theorem by Lemma 3.10. Note that for all $n \geq 0$ we have $b a c^{n} b \rightarrow_{\mathcal{R}} c^{n} b$ and $b a c^{n} b=b c^{n} a b \rightarrow_{\mathcal{R}}$ $b c^{n}$, but there is no $\alpha \leq n-1$ and no trace $w$ such that $c^{n} b \rightarrow{ }_{\overline{\mathcal{R}}}^{\leq \alpha} w$ and $b c^{n} \rightarrow{ }_{\mathcal{R}}^{\leq \alpha} w$. Hence there is no $\alpha \in \mathbb{N}$ such that $\mathcal{R}$ is $\alpha$-confluent.

We remark that the one-step rewrite graph of the trace rewriting system $\mathcal{R}$ from Theorem 3.11 is even not isomorphic to the one-step rewrite graph of an arbitrary term rewriting system. The reason is that Lemma 3.10 can be easily generalized to term rewriting systems, because also term rewriting systems have only finitely many critical pairs [60].

One might try to extend Theorem 3.8 into two directions: by allowing more rewriting systems or by considering more expressive logics. The rest of this section shows boundaries beyond which this programme cannot be carried out.

First we show that omitting the restriction type $(S) \equiv_{J}$ type $(T)$ for a scattered rewrite rule $(S, T)$ yields graphs with undecidable first-order theories. In order to see this let us call a permutation rewrite rule over $\mathbb{M}$ a pair $(S, T)$ with the same properties as a scattered rewrite rule except that type $(S) \equiv_{J}$ type $(T)$ need not hold. A rewrite step $s \rightarrow_{\rho} t$, where $\rho$ is a permutation rewrite rule, is defined exactly in the same way as it was done for scattered rewrite rules. Again a permutation rewriting system $\mathcal{P}$ over $\mathbb{M}$ is a finite set of permutation rewrite rules over $\mathbb{M}$, and the relation $\rightarrow_{\mathcal{P}}$ is defined as usual.

Theorem 3.12. There exists a fixed permutation rewriting system $\mathcal{R}$ over $\Sigma^{*}$ for some finite alphabet $\Sigma$ such that the first-order theory of the model $\left(\Sigma^{*},\left(\rightarrow_{\rho}\right)_{\rho \in \mathcal{R}}\right)$ is undecidable. Furthermore we may assume that for every variable $x$ that occurs in some $\rho \in \mathcal{R}$ we have $L(x)=\operatorname{alph}(x)^{*}$. 
Proof. By $[43,54]$ there exists a fixed semi-Thue system $\mathcal{S}$ over some $\Gamma^{*}$ with an undecidable word problem, i.e., it is undecidable for given words $s, t \in \Gamma^{*}$ whether or not $s \stackrel{*}{\leftrightarrow} \mathcal{S} t$, where $\stackrel{*}{\leftrightarrow}_{\mathcal{S}}$ denotes the transitive and symmetric closure of $\rightarrow \mathcal{S}$.

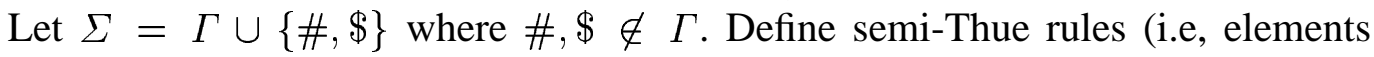
of a semi-Thue system) $\rho_{0}, \rho_{1}, \rho_{2}$, and $\rho_{a}$ for $a \in \Gamma$, by $\rho_{0}=(\$, \$), \rho_{1}=(\#, \$)$, $\rho_{2}=(\$ \#, \$ \#)$, and $\rho_{a}=(\$ a, \$)$ for $a \in \Gamma$. For a word $s=s_{1} s_{2} \cdots s_{m}$ with $s_{i} \in \Gamma$, let $\varphi_{s}$ denote the following formula with one free variable $w$ :

$$
\varphi_{s}(w) \equiv \neg\left(w \rightarrow_{\rho_{0}} w\right) \wedge \exists u_{0} \cdots \exists u_{m}\left\{\begin{array}{l}
w \rightarrow_{\rho_{1}} u_{0} \wedge \\
\bigwedge_{1 \leq \ell \leq m}\left(u_{\ell-1} \rightarrow_{\rho_{s_{\ell}}} u_{\ell}\right) \wedge \\
u_{m} \rightarrow_{\rho_{2}} u_{m}
\end{array}\right\}
$$

A word $w \in \Sigma^{*}$ satisfies this formula if and only if $w=w_{0} \# w_{1} \# \cdots w_{k-1} \# w_{k}$ with $w_{i} \in \Gamma^{*}$ and there exists $1 \leq j<k$ such that $w_{j}=s$. Next choose variables $x, y, x_{1}, y_{1}, x_{2}, y_{2} \in \Omega$ with $L(x)=L(y)=\Sigma^{*}$ and $L\left(x_{1}\right)=L\left(x_{2}\right)=L\left(y_{1}\right)=$ $L\left(y_{2}\right)=\Gamma^{*}$ and define the scattered rewrite rule $\rho_{\$}=\left(x \# x_{1} \# y_{1} \# y, x \$ x_{1} \$ y_{1} \$ y\right)$ and the permutation rewriting system

$$
\mathcal{P}=\left\{\left(x \$ x_{1} \ell y_{1} \$ x_{2} r y_{2} \$ y, x \$ x_{2} \ell y_{2} \$ x_{1} r y_{1} \$ y\right) \mid(\ell, r) \in \mathcal{S} \text { or }(r, \ell) \in \mathcal{S}\right\} .
$$

Then for given words $s, t \in \Gamma^{*}$ such that $s \neq t$ we have $s \stackrel{*}{\leftrightarrow} s t$ if and only if $\exists w\left\{\varphi_{s}(w) \wedge \varphi_{t}(w) \wedge \forall v\left(w \rightarrow_{\rho_{\S}} v \Rightarrow v \rightarrow_{\mathcal{P}} v\right)\right\}$. The important fact is that $\$ u \$ v \$ \rightarrow_{\mathcal{P}} \$ u \$ v \$$ for $u, v \in \Gamma^{*}$ if and only if $u \leftrightarrow_{\mathcal{S}} v$.

Next, we consider more expressive logics. The undecidability results for semi-Thue systems in Table 1 imply that we cannot generalize Corollary 3.9 to monadic secondorder logic. By the next theorem, also the decidability of the monadic second-order theory of the one-step rewrite graph of a prefix semi-Thue system [9], see Table 2, does not generalize to prefix rewriting systems over trace monoids (as defined in Section 3.2). In fact, already reachability is undecidable in these graphs:

Theorem 3.13. There exists a fixed prefix rewriting system $\mathcal{R}$ over a trace monoid $\mathbb{M}=\mathbb{M}(\Sigma, I)$ and a trace $t$ such that the following is undecidable:

INPUT: A trace $s \in \mathbb{M}$.

QUESTION: Does $s \stackrel{*}{\rightarrow}_{\mathcal{R}}$ t hold?

Proof. Let $\mathcal{C}$ be a universal deterministic 2-counter machine. Then $\mathcal{C}$ can be easily simulated by a prefix rewriting system $\mathcal{R}$ over $\{a, b\}^{*} \times\{c, d\}^{*}$ : the configuration $(q, m, n)$ of $\mathcal{C}$, where $q \in \mathbb{N}$ is the current state (we assume w.l.o.g. that the set of states of $\mathcal{C}$ is a finite subset of $\mathbb{N}$ ) and $m$ (resp. $n$ ) is the content of the first (resp. second) counter, is coded by the pair $\left(a^{q} b a^{m} b, c^{n} d\right)$. Let $q_{0}$ be the initial state of $\mathcal{C}$ and $q_{f}$ be the final state of $\mathcal{C}$. Hence, it is undecidable for given $m, n \in \mathbb{N}$ whether or not $\left(a^{q_{0}} b a^{m} b, c^{n} d\right) \stackrel{*}{\rightarrow}_{\mathcal{R}}\left(a^{q_{f}} b b, d\right)$.

If $\mathcal{R}$ is the prefix rewriting system from the above proof, then Theorem 3.13 implies that the model $\left(\mathbb{M}, \stackrel{*}{\rightarrow}_{\mathcal{R}},(t)_{t \in \mathbb{M}}\right)$ has an undecidable first-order theory (which cannot happen for prefix semi-Thue systems). 


\section{An excursion to word and trace equations}

In this section we will prove some results concerning equations in trace monoids. These results will be used in Section 5 where we prove Theorem 3.8. They state that certain equations (with recognizable constraints) are equivalent (in the model $\mathcal{M}$ ) to Boolean combinations of recognizable constraints. It is important to note that these equivalences are effective in the sense that starting from an equation (with recognizable constraints) that satisfies certain restrictions we can effectively construct an equivalent Boolean combination of recognizable constraints. This fact is not stated explicitly in the following lemmas, but it can always be checked easily. A similar remark applies to the results of Section 5.

Lemma 4.1. For $s, t \in \Sigma^{*}$, the set $\left\{u \in \Sigma^{*} \mid s u=u t\right\}$ is recognizable.

Proof. By [41, Prop. 1.3.4], we have $s u=u t$ if and only if there exist words $x$ and $y$ such that $s=x y, t=y x$, and $u \in x(y x)^{*}$. Thus, the set in question equals

$$
\bigcup\left\{x(y x)^{*} \mid s=x y, t=y x\right\} .
$$

Since $s$ and $t$ are finite words, this union is finite proving the lemma.

The next lemma generalizes the previous lemma to the case of several variables.

Lemma 4.2. Let $s_{1}, \ldots, s_{m+1}, t_{1}, \ldots, t_{m+1} \in \Sigma^{*}$. Then the formula

$$
s_{1} x_{1} s_{2} \cdots x_{m} s_{m+1}=t_{1} x_{1} t_{2} \cdots x_{m} t_{m+1}
$$

is equivalent in the model $\left(\Sigma^{*}, \circ,(a)_{a \in \Sigma},(K)_{K \in \mathrm{REC}\left(\Sigma^{*}\right)}\right)$ to a Boolean combination of formulae of the form $x_{i} \in L$ with $L \in \operatorname{REC}\left(\Sigma^{*}\right)$.

Proof. We prove the lemma by an induction on $m$. The case $m=0$ is trivial. So assume that $m>0$. If neither $s_{1}$ is a prefix of $t_{1}$ nor $t_{1}$ is a prefix of $s_{1}$ then (1) is equivalent to the truth value false. Now assume w.l.o.g. that $s_{1}=t_{1} s$. By cancelling $t_{1}$ we obtain the equation $s x_{1} s_{2} \cdots x_{m} s_{m+1}=x_{1} t_{2} \cdots x_{m} t_{m+1}$. If $s=\epsilon$ then we can also cancel the variable $x_{1}$ which allows us to apply the induction hypothesis. Now assume that $s \neq \epsilon$. We can guess the position in $t_{2} x_{2} \cdots t_{m} x_{m} t_{m+1}$ where $s x_{1}$ ends. More precisely, the word equation $s x_{1} s_{2} \cdots x_{m} s_{m+1}=x_{1} t_{2} \cdots x_{m} t_{m+1}$ is equivalent to the finite disjunction of all formulae of one of the following two forms:

$$
\begin{aligned}
- & s x_{1}=x_{1} t_{2} x_{2} \cdots t_{i-1} x_{i-1} t^{\prime} \wedge s_{2} x_{2} s_{3} \cdots x_{m} s_{m+1}=t^{\prime \prime} x_{i} t_{i+1} \cdots x_{m} t_{m+1} \text { where } \\
& 2 \leq i \leq m+1 \text { and } t_{i}=t^{\prime} t^{\prime \prime}\left(\text { i.e., } s x_{1} \text { ends in } t_{i}\right) \\
- & \exists x \exists y\left\{\begin{array}{l}
x_{i}=x y \wedge s x_{1}=x_{1} t_{2} \cdots x_{i-1} t_{i} x \wedge \\
s_{2} x_{2} s_{3} \cdots x_{m} s_{m+1}=y t_{i+1} x_{i+1} t_{i+2} \cdots x_{m} t_{m+1}
\end{array}\right\} \text { where } 2 \leq i \leq m, \text { and } \\
& \left.x \text { and } y \text { are new variables (i.e., } s x_{1} \text { ends in } x_{i} \text { where } i \geq 2 \text { because of } s \neq \epsilon\right) .
\end{aligned}
$$


It suffices to show that each of these formulae is equivalent to a Boolean combination of recognizable constraints. For this let us consider a formula of the second type, the first type can be dealt with similarly. If we substitute $x y$ for $x_{i}$ we obtain the formula

$$
\exists x \exists y\left\{\begin{array}{l}
x_{i}=x y \wedge s x_{1}=x_{1} t_{2} x_{2} \cdots t_{i-1} x_{i-1} t_{i} x \wedge \\
s_{2} x_{2} s_{3} \cdots x_{i-1} s_{i} x y s_{i+1} x_{i+1} \cdots s_{m} x_{m} s_{m+1}=y t_{i+1} x_{i+1} t_{i+2} \cdots x_{m} t_{m+1}
\end{array}\right\},
$$

see also the following picture.

\begin{tabular}{|l|l|l|l|l|l|l|l|l|l|l|l|}
\hline \multicolumn{4}{|c|}{$s$} & $x_{1}$ & $s_{2}$ & $x_{2}$ & $\cdots$ & $x_{m}$ & $s_{m+1}$ \\
\hline$x_{1}$ & $t_{2}$ & $\cdots$ & $t_{i}$ & $x$ & $y$ & $t_{i+1}$ & $\cdots$ & $x_{m}$ & $t_{m+1}$ \\
\hline \multicolumn{10}{|c|}{$x_{i}$} \\
\cline { 2 - 6 }
\end{tabular}

Note that for every solution of the equation $s x_{1}=x_{1} t_{2} \cdots x_{i-1} t_{i} x$, i.e., for every tuple $\left(u_{1}, u_{2}, \ldots, u_{i-1}, u\right)$ with $s u_{1}=u_{1} t_{2} \cdots u_{i-1} t_{i} u$, the length constraint $|s|=$ $\left|t_{2} u_{2} \cdots t_{i-1} u_{i-1} t_{i} u\right|$ holds. But there are only finitely many tuples $\left(u_{2}, \ldots, u_{i-1}, u\right)$ that satisfy this constraint. Hence we can take the finite disjunction over all tuples $\left(u_{2}, \ldots, u_{i-1}, u\right)$ with $|s|=\left|t_{2} u_{2} \cdots t_{i-1} u_{i-1} t_{i} u\right|$ where each single disjunctive subformula results from substituting in (2) $u_{i}$ for $x_{i}$ and $u$ for $x$. Let $\left(u_{2}, \ldots, u_{i-1}, u\right)$ be one of these tuples and define $v=t_{2} u_{2} \cdots t_{i-1} u_{i-1} t_{i} u$ and $w=s_{2} u_{2} \cdots s_{i-1} u_{i-1} s_{i} u$. It suffices to show that the formula

$$
\exists y\left\{\begin{array}{l}
x_{i}=u y \wedge s x_{1}=x_{1} v \wedge \bigwedge_{2 \leq j \leq i-1} x_{j} \in\left\{u_{j}\right\} \wedge \\
w y s_{i+1} x_{i+1} \cdots s_{m} x_{m} s_{m+1}=y t_{i+1} x_{i+1} \cdots t_{m} x_{m} t_{m+1}
\end{array}\right\}
$$

is equivalent to a Boolean combination of recognizable constraints. By Lemma 4.1 we can replace the equation $s x_{1}=x_{1} v$ by a formula of the form $x_{1} \in L_{1}$ where $L_{1} \in \operatorname{REC}\left(\Sigma^{*}\right)$. Furthermore the induction hypothesis ${ }^{2}$ implies that the equation

$$
w y s_{i+1} x_{i+1} \cdots s_{m} x_{m} s_{m+1}=y t_{i+1} x_{i+1} \cdots t_{m} x_{m} t_{m+1}
$$

is equivalent to a finite disjunction of formulae of the form $y \in K \wedge \bigwedge_{i+1 \leq j \leq m} x_{j} \in L_{j}$ where $K, L_{i+1}, \ldots, L_{m} \in \operatorname{REC}\left(\Sigma^{*}\right)$. In this way we obtain a finite disjunction of formulae of the form

$$
\exists y\left\{x_{i}=u y \wedge x_{1} \in L_{1} \wedge \bigwedge_{2 \leq j \leq i-1} x_{j} \in\left\{u_{j}\right\} \wedge y \in K \wedge \bigwedge_{i+1 \leq j \leq m} x_{j} \in L_{j}\right\} .
$$

But this formula is equivalent to the formula

$$
x_{1} \in L_{1} \wedge \bigwedge_{2 \leq j \leq i-1} x_{j} \in\left\{u_{j}\right\} \wedge x_{i} \in u K \wedge \bigwedge_{i+1 \leq j \leq m} x_{j} \in L_{j} .
$$

This concludes the proof of the lemma.

\footnotetext{
${ }^{2}$ Note that because of $i \geq 2$, (3) contains $m-i+1<m$ many variables.
} 
Next we will generalize Lemma 4.2 from words to traces. For this we need the following simple lemma.

Lemma 4.3. Let $\left(\Gamma_{1}, \ldots, \Gamma_{n}\right)$ be a clique covering of the dependence alphabet $(\Sigma, D)$. For $1 \leq i \leq n$ let $L_{i} \in \operatorname{REC}\left(\Gamma_{i}^{*}\right)$. Then the set of all traces $u \in \mathbb{M}$ such that $\pi_{i}(u) \in L_{i}$ for $1 \leq i \leq n$ is recognizable.

Proof. We define a morphism $\pi: \mathbb{M} \rightarrow \Gamma_{1}^{*} \times \cdots \times \Gamma_{n}^{*}$ by $\pi(u)=\left(\pi_{1}(u), \ldots, \pi_{n}(u)\right)$. Since $L_{1} \times \cdots \times L_{n} \in \operatorname{REC}\left(\Gamma_{1}^{*} \times \cdots \times \Gamma_{n}^{*}\right)$ and recognizable languages are closed under inverse morphisms [23], the language $\left\{u \in \mathbb{M} \mid \pi_{1}(u) \in L_{1}, \ldots, \pi_{n}(u) \in L_{n}\right\}$ is recognizable.

Lemma 4.4. Let $S\left(x_{1}, \ldots, x_{m}\right)$ and $T\left(x_{1}, \ldots, x_{m}\right)$ be patterns such that $\operatorname{type}(S) \equiv_{J}$ type $(T) .{ }^{3}$ Then the formula

$$
\bigwedge_{1 \leq i \leq m} x_{i} \in L\left(x_{i}\right) \wedge S\left(x_{1}, \ldots, x_{m}\right)=T\left(x_{1}, \ldots, x_{m}\right)
$$

is equivalent in $\mathcal{M}$ to a Boolean combination of formulae $x_{i} \in L$ with $L \in R E C(\mathbb{M})$.

Proof. Let $\left(\Gamma_{1}, \ldots, \Gamma_{n}\right)$ be an arbitrary clique covering of the dependence alphabet $(\Sigma, D)$. For all $i \in\{1, \ldots, m\}$ and all $j \in\{1, \ldots, n\}$ let $x_{i, j}$ be a new variable which will represent the projection of $x_{i}$ onto the clique $\Gamma_{j}$. Since $x_{i}$ is restricted to the set $L\left(x_{i}\right) \subseteq \operatorname{alph}\left(x_{i}\right)^{*}$, we can replace the variable $x_{i, j}$ by the empty word $\epsilon$ in case $\operatorname{alph}\left(x_{i}\right) \cap \Gamma_{j}=\emptyset$. For this we define for every $j \in\{1, \ldots, n\}$ a substitution $\vartheta_{j}$ by

$$
\vartheta_{j}(a)=\pi_{j}(a) \quad \text { for } a \in \Sigma \quad \text { and } \quad \vartheta_{j}\left(x_{i}\right)= \begin{cases}\epsilon & \text { if } \operatorname{alph}\left(x_{i}\right) \cap \Gamma_{j}=\emptyset \\ x_{i, j} & \text { otherwise. }\end{cases}
$$

Thus, the pattern $\vartheta_{j}(S)$ represents the projection of the pattern $S$ onto the clique $\Gamma_{j}$. We claim that type $\left(\vartheta_{j}(S)\right)=\operatorname{type}\left(\vartheta_{j}(T)\right)$ for all $j \in\{1, \ldots, n\}$. Since type $(S) \equiv_{J}$ type $(T)$, it suffices to prove that $\vartheta_{j}(y z)=\vartheta_{j}(z y)$ for variables $y, z \in\left\{x_{1}, \ldots, x_{m}\right\}$ with $(y, z) \in J$. Let $(y, z) \in J$. If $\Gamma_{j}$ is a clique then we cannot have $\operatorname{alph}(y) \cap \Gamma_{j} \neq$ $\emptyset \neq \operatorname{alph}(z) \cap \Gamma_{j}$, since this contradicts $(y, z) \in J$. Thus either $\vartheta_{j}(y)=\epsilon$ or $\vartheta_{j}(z)=\epsilon$, which implies $\vartheta_{j}(y z)=\vartheta_{j}(z y)$.

By Lemma 3.3, the equation $S\left(x_{1}, \ldots, x_{m}\right)=T\left(x_{1}, \ldots, x_{m}\right)$ is satisfied by traces $x_{i} \in L\left(x_{i}\right) \subseteq \operatorname{alph}\left(x_{i}\right)^{*}$ for $1 \leq i \leq m$ if and only if their projections $x_{i, j}=\pi_{j}\left(x_{i}\right) \in$ $\left(\operatorname{alph}\left(x_{i}\right) \cap \Gamma_{j}\right)^{*}$ satisfy the equations $\vartheta_{j}(S)=\vartheta_{j}(T)$ for all $1 \leq j \leq n$. But since $\Gamma_{j}$ is a clique of $(\Sigma, D)$ this holds if and only if the projections $x_{i, j}$ satisfy a Boolean combination of recognizable constraints by Lemma 4.2. By transforming this Boolean combination into disjunctive normal form and using the closure of $\operatorname{REC}\left(\Gamma_{j}^{*}\right)$ under Boolean operations, we obtain an equivalent finite disjunction of conjunctions of the form

$$
\bigwedge_{1 \leq i \leq m} x_{i, j} \in L_{i, j}
$$

\footnotetext{
${ }^{3}$ Note that this means that $(S, T) \in \mathbb{S}$.
} 
where $L_{i, j} \in \operatorname{REC}\left(\Gamma_{j}^{*}\right)$. Hence (4) is satisfied for traces $x_{1}, \ldots, x_{m} \in \mathbb{M}$ if and only if $x_{1}, \ldots, x_{m}$ satisfy a finite disjunction of formulae of the form

$$
\bigwedge_{1 \leq i \leq m} x_{i} \in\left\{u \in \mathbb{M} \mid \pi_{1}(u) \in L_{i, 1}, \ldots, \pi_{n}(u) \in L_{i, n}\right\} \cap L\left(x_{i}\right)
$$

with $L_{i, j} \in \operatorname{REC}\left(\Gamma_{j}^{*}\right)$. By Lemma 4.3 the sets appearing in this formula are recognizable which concludes the proof.

\section{Decidability of scattered rewriting}

In this section we will prove Theorem 3.8. A remark similar to that at the beginning of the previous section applies: all existence and equivalence results in this section are effective. This fact is not stated explicitly in the following lemmas, but it can always be checked easily.

\subsection{Reduction to local properties}

The main tool in this section is Gaifman's locality theorem for first-order logic [25]. We will only need the special case of Gaifman's Theorem where the signature contains only unary and binary relation symbols which makes the definitions a little bit easier. Let us consider a model of the form $\mathcal{A}=(A, U, B)$ where $U$ is a set of unary relations, and $B$ is a set of binary relations. For $a, b \in A$ we define $d_{\mathcal{A}}(a, b)$ as the length of a shortest undirected path in the graph $\left(A, \bigcup_{E \in B} E\right)$ from $a$ to $b$. For $r \geq 0$ and $a \in A$, the $r$-sphere $S(r, a)$ around $a$ is $S(r, a)=\left\{b \in A \mid d_{\mathcal{A}}(a, b) \leq r\right\}$. There exists a first-order formula over the signature of $\mathcal{A}$ with two free variables that expresses the fact that $d_{\mathcal{A}}(x, y) \leq r$, i.e., the $r$-sphere around $a$ is definable. Now let $\varphi$ be a first-order formula over the signature of $\mathcal{A}$ and let $x$ be a variable. Then the firstorder formula $\varphi^{S(r, x)}$ results from $\varphi$ by relativizing all quantifiers to $S(r, x)$. It can be defined inductively, for instance $\left(\varphi_{1} \wedge \varphi_{2}\right)^{S(r, x)} \equiv \varphi_{1}^{S(r, x)} \wedge \varphi_{2}^{S(r, x)},(\exists y \varphi)^{S(r, x)} \equiv$ $\exists y\left\{d(x, y) \leq r \wedge \varphi^{S(r, x)}\right\}$ (where $y$ has to be renamed into a fresh variable if $y=$ $x)$, and $P\left(x_{1}, \ldots, x_{n}\right)^{S(r, x)} \equiv P\left(x_{1}, \ldots, x_{n}\right)$ for atomic formulae. It is allowed that the formula $\varphi$ contains the variable $x$ free. Moreover, the formula $\varphi^{S(r, x)}$ certainly contains $x$ free if $\varphi$ contains at least one quantifier. Now Gaifman's Theorem states the following [25].

Theorem 5.1. Let $\varphi$ be a first-order sentence over the signature of the model $\mathcal{A}$. Then $\varphi$ is logically equivalent to a Boolean combination of sentences of the form

$$
\exists x_{1} \cdots \exists x_{m}\left\{\bigwedge_{1 \leq i<j \leq m} d\left(x_{i}, x_{j}\right)>2 r \wedge \bigwedge_{1 \leq i \leq m} \psi\left(x_{i}\right)^{S\left(r, x_{i}\right)}\right\}
$$

where $\psi(x)$ is a first-order formula over the signature of $\mathcal{A}$ that contains at most $x$ free and $\psi\left(x_{i}\right)$ results from $\psi(x)$ by replacing every free occurrence of $x$ by $x_{i}$. 
Since $\mathcal{M}^{\text {red }}$ contains all scattered rewrite rules, we get $d_{\mathcal{M}^{\text {red }}}(u, v) \leq 1$ for any two traces $u$ and $v$. Hence for the treatment of the structure $\mathcal{M}^{\text {red }}$, Theorem 5.1 is of no immediate use. But any first-order sentence $\varphi$ over the signature of $\mathcal{M}^{\text {red }}$ mentions only finitely many scattered rewrite relations $\rightarrow_{\rho}$. Hence, $\mathcal{M}^{\text {red }} \models \varphi$ if and only if $\mathcal{M}_{\mathcal{R}}^{\text {red }} \models \varphi$ where $\mathcal{R} \subseteq \mathbb{S}$ is finite and contains the set of rewrite rules mentioned in $\varphi$. Thus, in order to prove the decidability of the first-order theory of $\mathcal{M}^{\text {red }}$, it suffices to prove the decidability of the first-order theory of $\mathcal{M}_{\mathcal{R}}^{\text {red }}$ for any finite set of rewrite rules $\mathcal{R}$. Therefore, for our further considerations, we fix some finite set $\mathcal{R} \subseteq \mathbb{S}$ of scattered rewrite rules.

In order to use Gaifman's Theorem for decidability purposes, we will need a "useful" description of the set of all traces $t \in \mathbb{M}$ with $\mathcal{M}_{\mathcal{R}}^{\text {red }} \models \psi(x)^{S(r, x)}(t)$. We will show that this set is recognizable and that it is indeed a "useful" description (cf. Section 5.4). The following two sections prepare the proof of the recognizability.

\subsection{Reduction to 1-spheres}

The aim of this section is to show that, by enlarging the set $\mathcal{R}$, it suffices to restrict to the case $r=1$ in Theorem 5.1. For the rest of Section 5, $\bar{x}$ (resp. $\bar{y}$ ) will always denote the repetition-free sequence $x_{1}, \ldots, x_{m}$ (resp. $y_{1}, \ldots, y_{n}$ ) of variables from $\Omega$. We assume that $\bar{x}$ and $\bar{y}$ do not contain common variables. Furthermore we use the formula $\bar{x} \in L(\bar{x})$ as an abbreviation for the formula $\bigwedge_{1 \leq i \leq m} x_{i} \in L\left(x_{i}\right)$, and similarly for $\bar{y}$. The following lemma follows immediately from Lemma 3.2.

Lemma 5.2. Let $T(\bar{x})$ and $U(\bar{y})$ be patterns with type $(T)=x_{1} \cdots x_{m}$ and $\operatorname{type}(U)=$ $y_{1} \cdots y_{n}$. Then the equation $T(\bar{x})=U(\bar{y})$ is equivalent in $\mathcal{M}$ to a finite disjunction of formulae of the form

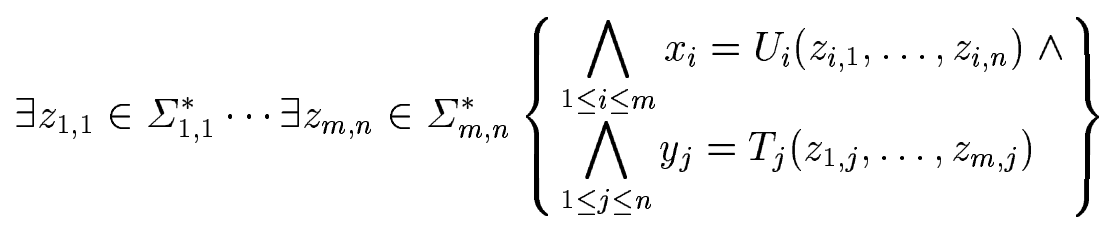

where type $\left(T_{j}\right)=z_{1, j} z_{2, j} \cdots z_{m, j}$, type $\left(U_{i}\right)=z_{i, 1} z_{i, 2} \cdots z_{i, n}$, and the alphabets $\Sigma_{i, j}$ satisfy $\Sigma_{i, j} \times \Sigma_{k, \ell} \subseteq I$ whenever $1 \leq i<k \leq m$ and $n \geq j>\ell \geq 1$.

Lemma 5.3. Let $S(\bar{x})$ be a pattern, and let $L \in \operatorname{REC}(\mathbb{M})$. Then the formula $x=$ $S(\bar{x}) \wedge x \in L$ is equivalent in $\mathcal{M}$ to a finite disjunction of formulae of the form

$$
x=S(\bar{x}) \wedge \bigwedge_{1 \leq i \leq m} x_{i} \in L_{i}
$$

where $L_{i} \in \operatorname{REC}(\mathbb{M})$ and $\operatorname{alph}\left(L_{i}\right) \subseteq \operatorname{alph}(L)$.

Proof. By renaming the variables if necessary, we may assume w.l.o.g. that $S=$ $x_{1} s_{1} x_{2} \cdots s_{m-1} x_{m}$. Let $h: \mathbb{M} \rightarrow Q$ be a morphism into a finite monoid $Q$ and let 
$F \subseteq Q$ such that $L=h^{-1}(F)$. In order to construct the desired formula let us take a finite disjunction over all sequences $q_{1}, q_{2}, \ldots, q_{m}$ of elements from $Q$ such that $q_{1} h\left(s_{1}\right) q_{2} \cdots h\left(s_{m-1}\right) q_{m} \in F$. For such a sequence we write down the formula

$$
x=S(\bar{x}) \wedge \bigwedge_{1 \leq i \leq m} x_{i} \in h^{-1}\left(q_{i}\right) .
$$

It is easy to see that the resulting disjunction is equivalent in $\mathcal{M}$ to our original formula. Note that every trace $t \in h^{-1}\left(q_{i}\right)$ is a factor of some $s \in L$. Thus alph $\left(h^{-1}\left(q_{i}\right)\right) \subseteq$ $\operatorname{alph}(L)$.

Let $\rho_{1}$ and $\rho_{2}$ be scattered rewrite rules. Then the following lemma implies easily that there exists a finite scattered rewriting system $\mathcal{P}$ such that $x \rightarrow_{\mathcal{P}} y$ is equivalent to $\exists z: x \rightarrow_{\rho_{1}} z \rightarrow_{\rho_{2}} y$, for all traces $x$ and $y .{ }^{4}$ In general, $\mathcal{P}$ consists of more than just one rule. The lemma will allow us to describe the $r$-sphere around a trace $x$ as the 1-sphere of another, larger, but still finite, scattered rewriting system (cf. Lemma 5.5).

Lemma 5.4. Let $(S(\bar{x}), T(\bar{x})),(U(\bar{y}), V(\bar{y})) \in \mathbb{S}$. The formula

$$
x=S(\bar{x}) \wedge \bar{x} \in L(\bar{x}) \wedge T(\bar{x})=U(\bar{y}) \wedge y=V(\bar{y}) \wedge \bar{y} \in L(\bar{y})
$$

is equivalent in $\mathcal{M}$ to a finite disjunction of formulae of the form

$$
\exists z_{1,1} \in L\left(z_{1,1}\right) \cdots \exists z_{m, n} \in L\left(z_{m, n}\right)\left\{\begin{array}{l}
x=P\left(z_{1,1}, \ldots, z_{m, n}\right) \wedge \\
\bigwedge_{1 \leq i \leq m} x_{i}=U_{i}\left(z_{i, 1}, \ldots, z_{i, n}\right) \wedge \\
\bigwedge_{1 \leq j \leq n} y_{j}=T_{j}\left(z_{1, j}, \ldots, z_{m, j}\right) \wedge \\
y=R\left(z_{1,1}, \ldots, z_{m, n}\right)
\end{array}\right\}
$$

where $U_{i}\left(z_{i, 1}, \ldots, z_{i, n}\right)$ and $T_{j}\left(z_{1, j}, \ldots, z_{m, j}\right)$ are patterns and $(P, R)$ is a scattered rewrite rule over the variables $z_{1,1}, \ldots, z_{m, n}$.

Proof. By renaming the variables if necessary, we may assume w.l.o.g. that type $(T)=$ $x_{1} \cdots x_{m}$ and type $(U)=y_{1} \cdots y_{n}$. By Lemma 5.2 the formula (5) is equivalent in $\mathcal{M}$ to a finite disjunction of formulae of the form

$$
\exists z_{1,1} \in \Sigma_{1,1}^{*} \ldots \exists z_{m, n} \in \Sigma_{m, n}^{*}\left\{\begin{array}{l}
x=S(\bar{x}) \wedge \bar{x} \in L(\bar{x}) \wedge \\
\bigwedge_{1 \leq i \leq m} x_{i}=U_{i}\left(z_{i, 1}, \ldots, z_{i, n}\right) \wedge \\
\bigwedge_{1 \leq j \leq n} y_{j}=T_{j}\left(z_{1, j}, \ldots, z_{m, j}\right) \wedge \\
y=V(\bar{y}) \wedge \bar{y} \in L(\bar{y})
\end{array}\right\}
$$

\footnotetext{
${ }^{4}$ The corresponding statement for ordinary trace rewriting systems is not true. This is our main reason for generalizing trace rewriting systems to scattered rewriting systems.
} 
where type $\left(T_{j}\right)=z_{1, j} z_{2, j} \cdots z_{m, j}$, type $\left(U_{i}\right)=z_{i, 1} z_{i, 2} \cdots z_{i, n}$, and the alphabets $\Sigma_{i, j}$ satisfy $\Sigma_{i, j} \times \Sigma_{k, \ell} \subseteq I$ whenever $1 \leq i<k \leq m$ and $n \geq j>\ell \geq 1$. By Lemma 5.3 we can replace the subformulae $x_{i}=U_{i}\left(z_{i, 1}, \ldots, z_{i, n}\right) \wedge x_{i} \in L\left(x_{i}\right)$ in (6) by a finite disjunction of formulae of the form

$$
x_{i}=U_{i}\left(z_{i, 1}, \ldots, z_{i, n}\right) \wedge \bigwedge_{1 \leq j \leq n} z_{i, j} \in K_{i, j}
$$

where $K_{i, j} \in \operatorname{REC}(\mathbb{M})$ and $\operatorname{alph}\left(K_{i, j}\right) \subseteq \operatorname{alph}\left(x_{i}\right)$. Similarly, the subformulae $y_{j}=$ $T_{j}\left(z_{1, j}, \ldots, z_{m, j}\right) \wedge y_{j} \in L\left(y_{j}\right)$ can be replaced in (6) by a finite disjunction of formulae of the form

$$
y_{j}=T_{j}\left(z_{1, j}, \ldots, z_{m, j}\right) \wedge \bigwedge_{1 \leq i \leq m} z_{i, j} \in L_{i, j}
$$

where $L_{i, j} \in \operatorname{REC}(\mathbb{M})$ and $\operatorname{alph}\left(L_{i, j}\right) \subseteq \operatorname{alph}\left(y_{j}\right)$. Altogether we obtain for the variable $z_{i, j}$ the recognizable constraint $z_{i, j} \in \Sigma_{i, j}^{*} \cap K_{i, j} \cap L_{i, j}$. We may w.l.o.g. assume that the variable $z_{i, j}$ was chosen such that $L\left(z_{i, j}\right)=\Sigma_{i, j}^{*} \cap K_{i, j} \cap L_{i, j}$. By the independence properties of the alphabets $\Sigma_{i, j}$ that followed from Lemma 5.2, and by the inclusions $\operatorname{alph}\left(K_{i, j}\right) \subseteq \operatorname{alph}\left(x_{i}\right)$ and $\operatorname{alph}\left(L_{i, j}\right) \subseteq \operatorname{alph}\left(y_{j}\right)$, this implies that $\left(z_{i, j}, z_{k, \ell}\right) \in J$ whenever $(i<k$ and $j>\ell)$ or $\left(x_{i}, x_{k}\right) \in J$ or $\left(y_{j}, y_{\ell}\right) \in J$.

Finally we may replace the pattern $S$ by the pattern $P=S\left[x_{i} / U_{i}\right]$, which results from $S$ by replacing for all $i$ the variable $x_{i}$ by the pattern $U_{i}$. Similarly, we may replace the pattern $V$ by the pattern $R=V\left[y_{j} / T_{j}\right]$. Then syntactically, the resulting formula has the desired form. It remains to show that type $(P) \equiv_{J}$ type $(R)$. Since $\left(z_{i, j}, z_{k, \ell}\right) \in$ $J$ if $\left(x_{i}, x_{k}\right) \in J$, we have $\left(\operatorname{type}\left(U_{i}\right)\right.$, type $\left.\left(U_{k}\right)\right) \in J$ whenever $\left(x_{i}, x_{k}\right) \in J$. Since $\operatorname{type}(S) \equiv_{J} \operatorname{type}(T)=x_{1} \cdots x_{m}$ it follows that

$$
\operatorname{type}(P) \equiv_{J} \operatorname{type}\left(U_{1}\right) \cdots \operatorname{type}\left(U_{m}\right)=\left(z_{1,1} \cdots z_{1, n}\right) \cdots\left(z_{m, 1} \cdots z_{m, n}\right) .
$$

Analogously we obtain

$$
\operatorname{type}(R) \equiv_{J} \operatorname{type}\left(T_{1}\right) \cdots \operatorname{type}\left(T_{n}\right)=\left(z_{1,1} \cdots z_{m, 1}\right) \cdots\left(z_{1, n} \cdots z_{m, n}\right) .
$$

Finally, since $\left(z_{i, j}, z_{k, \ell}\right) \in J$ if $i<k$ and $j>\ell$, we have

$$
\left(z_{1,1} \cdots z_{1, n}\right) \cdots\left(z_{m, 1} \cdots z_{m, n}\right) \equiv_{J}\left(z_{1,1} \cdots z_{m, 1}\right) \cdots\left(z_{1, n} \cdots z_{m, n}\right),
$$

which implies type $(P) \equiv_{J}$ type $(R)$.

Lemma 5.5. For $r \geq 0$ and a scattered rewriting system $\mathcal{R}$, there exists a scattered rewriting system $\mathcal{R}_{r}$ over $\mathbb{M}$ such that, for all $s, t \in \mathbb{M}$, we have $d_{\mathcal{M}_{\mathcal{R}}^{\text {red }}}(s, t) \leq r$ if and only if $s \rightarrow_{\mathcal{R}_{r}} t$.

Proof. Let $(S(\bar{x}), T(\bar{x})),(U(\bar{y}), V(\bar{y})) \in \mathbb{S}$. The formula $\exists z: x \rightarrow_{(S, T)} z \rightarrow_{(U, V)} y$ is equivalent to the formula

$$
\exists \bar{x} \exists \bar{y}\{x=S(\bar{x}) \wedge \bar{x} \in L(\bar{x}) \wedge T(\bar{x})=U(\bar{y}) \wedge y=V(\bar{y}) \wedge \bar{y} \in L(\bar{y})\},
$$


which is the formula (5) from Lemma 5.4, except that the variables $x_{i}$ and $y_{j}$ are quantified existentially. Hence, by Lemma 5.4 it is equivalent in $\mathcal{M}$ to a finite disjunction of formulae of the form

$$
\exists \bar{x} \exists \bar{y} \exists z_{1,1} \in L\left(z_{1,1}\right) \cdots \exists z_{m, n} \in L\left(z_{m, n}\right)\left\{\begin{array}{l}
x=P\left(z_{1,1}, \ldots, z_{m, n}\right) \wedge \\
\bigwedge_{1 \leq i \leq m} x_{i}=U_{i}\left(z_{i, 1}, \ldots, z_{i, n}\right) \wedge \\
\bigwedge_{1 \leq j \leq n} y_{j}=T_{j}\left(z_{1, j}, \ldots, z_{m, j}\right) \wedge \\
y=R\left(z_{1,1}, \ldots, z_{m, n}\right)
\end{array}\right\} .
$$

Any of those is, in $\mathcal{M}$, equivalent to

$$
\exists z_{1,1} \in L\left(z_{1,1}\right) \cdots \exists z_{m, n} \in L\left(z_{m, n}\right)\left\{\begin{array}{l}
x=P\left(z_{1,1}, \ldots, z_{m, n}\right) \wedge \\
y=R\left(z_{1,1}, \ldots, z_{m, n}\right)
\end{array}\right\},
$$

i.e., $x \rightarrow_{(P, R)} y$ where $(P, R) \in \mathbb{S}$.

Now we can prove the lemma inductively. Let $\mathcal{R}_{0}=\{(x, x)\}$, where $L(x)=\mathbb{M}$. Assume that $\mathcal{R}_{r-1}$ is already defined. Define the scattered rewriting system $\mathcal{S}=\mathcal{R} \cup$ $\{(S, R) \mid(R, S) \in \mathcal{R}\}$. Then $d_{\mathcal{M}_{\mathcal{R}}^{\text {red }}}(x, y) \leq r$ if and only if

$$
x \rightarrow_{\mathcal{R}_{r-1}} y \vee \exists z: x \rightarrow_{\mathcal{R}_{r-1}} z \rightarrow_{\mathcal{S}} y .
$$

From the discussion in the previous paragraph it follows that $\exists z: x \rightarrow_{\mathcal{R}_{r-1}} z \rightarrow_{\mathcal{S}} y$ is equivalent in $\mathcal{M}$ to $x \rightarrow_{\mathcal{S}^{\prime}} y$ for some scattered rewriting system $\mathcal{S}^{\prime}$. Thus, we can define $\mathcal{R}_{r}=\mathcal{R}_{r-1} \cup \mathcal{S}^{\prime}$.

Before we proceed let us first state the following simple consequence of Lemma 4.4 and Lemma 5.4, which will be useful in the next section.

Lemma 5.6. Let $(S(\bar{x}), T(\bar{x})),(V(\bar{y}), U(\bar{y})) \in \mathbb{S}$. The formula

$$
\bar{x} \in L(\bar{x}) \wedge S(\bar{x}) \rightarrow_{(V, U)} T(\bar{x})
$$

is equivalent in $\mathcal{M}$ to a Boolean combination of formulae $x_{i} \in L$ with $L \in \operatorname{REC}(\mathbb{M})$.

Proof. First note that (7) is equivalent in $\mathcal{M}$ to the formula

$$
\exists x \exists \bar{y}\{x=S(\bar{x}) \wedge \bar{x} \in L(\bar{x}) \wedge T(\bar{x})=U(\bar{y}) \wedge x=V(\bar{y}) \wedge \bar{y} \in L(\bar{y})\} .
$$

Similarly to the proof of Lemma 5.5 we can apply Lemma 5.4 to this formula (with $x=y$ in Lemma 5.4). We obtain an equivalent finite disjunction of formulae of the form

$$
\exists z_{1,1} \in L\left(z_{1,1}\right) \cdots \exists z_{m, n} \in L\left(z_{m, n}\right)\left\{\begin{array}{l}
P\left(z_{1,1}, \ldots, z_{m, n}\right)=R\left(z_{1,1}, \ldots, z_{m, n}\right) \wedge \\
\bigwedge_{1 \leq i \leq m} x_{i}=U_{i}\left(z_{i, 1}, \ldots, z_{i, n}\right)
\end{array}\right\}
$$


where type $(P) \equiv_{J}$ type $(R)$. Hence, by Lemma 4.4 this formula is equivalent in $\mathcal{M}$ to a finite disjunction of formulae of the form

$$
\exists z_{1,1} \in L\left(z_{1,1}\right) \cdots \exists z_{m, n} \in L\left(z_{m, n}\right) \bigwedge_{1 \leq i \leq m}\left(x_{i}=U_{i}\left(z_{i, 1}, \ldots, z_{i, n}\right) \wedge \bigwedge_{1 \leq j \leq n} z_{i, j} \in L_{i, j}\right)
$$

where $L_{i, j} \in \operatorname{REC}(\mathbb{M})$. Since the patterns $U_{i}$ and $U_{j}$ are defined over disjoint sets of variables for $i \neq j$, this formula is equivalent to

$$
\bigwedge_{1 \leq i \leq m} x_{i} \in\left\{U_{i}\left(u_{1}, \ldots, u_{n}\right) \mid u_{1} \in L_{i, 1} \cap L\left(z_{i, 1}\right), \ldots, u_{n} \in L_{i, n} \cap L\left(z_{i, n}\right)\right\}
$$

By the closure properties of $\operatorname{REC}(\mathbb{M})$ and since in every pattern $U_{i}$ each variable occurs at most once, the sets in the formula above are recognizable.

\subsection{Internalizing the 1-sphere}

As a major tool in our further considerations we will use monadic second-order logic (MSO logic) over dependence graphs. Formulae in this logic are interpreted over dependence graphs $(V, E, \lambda)$ of traces. This logic has first-order variables $x, y, z, \ldots$ ranging over elements of $V$ and second-order variables $X, Y, Z, \ldots$ ranging over subsets of $V$. Atomic formulae are of the form $Q_{a}(x), x \preceq y$, and $x \in X$ where $x$ and $y$ are first-order variables, $X$ is a second-order variable, and $Q_{a}$ is a unary relation symbol for every $a \in \Sigma$. The interpretation of $Q_{a}(x)$ is $\lambda(x)=a$ whereas $x \preceq y$ is interpreted as $(x, y) \in E^{*}$. From atomic formulae, MSO-formulae are constructed using Boolean connectives and quantification over first-order and second-order variables. For an MSO-formula $\psi$ and a trace $t \in \mathbb{M}$ we write $D_{t} \models \psi$ if the dependence graph $D_{t}$ satisfies the formula $\psi$.

We use the following terminology in order not to get confused by the different types of variables we have to deal with. First-order variables that range over elements of the model $\mathcal{M}$ are called external first-order variables. Second-order variables that range over subsets of an underlying dependence graph are called internal second-order variables. Internal first-order variables that range over single nodes of the dependence graph will not occur very much in our further considerations. Similarly, first-order formulae that speak about the infinite structure $\mathcal{M}$ (consisting of all traces) will be called external first-order formulae, whereas MSO-formulae that are interpreted over a single (finite) dependence graph are called internal MSO-formulae.

Thomas has shown in [62] that MSO logic precisely characterizes recognizable trace languages:

Theorem 5.7. A set $L \subseteq \mathbb{M}$ is recognizable if and only if there exists an MSOsentence $\Psi$ such that $L=\left\{t \in \mathbb{M} \mid D_{t}=\Psi\right\}$.

This result has been extended to other logics $[62,19]$ and to infinite traces [22]. 
We will need the following internal MSO-formulae. The MSO-formula $\operatorname{conv}(X)$ defined by

$$
\operatorname{conv}(X) \equiv \forall x \forall y \forall z\{(x, z \in X \wedge x \preceq y \preceq z) \rightarrow y \in X\}
$$

holds if and only if $X$ is a convex subset of the dependence graph. Recall that if $X$ is convex then the restricted dependence graph $D_{t} \uparrow_{X}$ is isomorphic to a dependence graph $D_{u}$ for a unique trace $u \in \mathbb{M}$. In the following, $D_{t} \uparrow_{X}$ will be also used in order to denote this trace $u$. For a pattern $S=x_{1} s_{1} x_{2} \cdots s_{m-1} x_{m}$ we define an MSO-formula

$$
\operatorname{fact}_{S}\left(X, X_{1}, \ldots, X_{m}\right)
$$

which expresses for a dependence graph $D_{t}=(V, E, \lambda)$ the following facts:

- $X, X_{1}, \ldots, X_{m}$ are convex and $D_{t} \uparrow_{X_{i}} \in L\left(x_{i}\right)$ for $1 \leq i \leq m$.

- There exist convex sets $Z_{1}, \ldots, Z_{m-1} \subseteq X$ of $D_{t}$ such that the trace represented by $D_{t} \uparrow_{Z_{i}}$ is $s_{i}$ and that the sequence $X_{1}, Z_{1}, X_{2}, Z_{2}, \ldots, Z_{m-1}, X_{m}$ is a factorization of $D_{t} \uparrow_{X}$ (according to the definition before Lemma 3.1).

Note that both points can be expressed in MSO logic. Finally we will use in MSOformulae several times a constant $V$ which denotes the whole set of nodes of the underlying dependence graph. Of course this constant can be easily defined in MSO logic.

Let $r \in \mathbb{N}$, let $\varphi$ be an external first-order formula over the signature of the model $\mathcal{M}_{\mathcal{R}}^{\text {red }}$ and let $x$ be a variable. By induction on the structure of $\varphi$ we will construct an internal monadic second-order sentence $\operatorname{int}(\varphi)$ such that, for $t \in \mathbb{M}$, we obtain $\mathcal{M} \models \varphi^{S(r, x)}(t)$ if and only if $D_{t} \models \operatorname{int}(\varphi)$. Thus an external first-order property of the $r$-sphere around a trace $t$ will be translated into an internal property of the dependence graph of the central trace $t$. Due to Lemma 5.5 we may, by replacing the system $\mathcal{R}$ by $\mathcal{R}_{r}$, restrict ourselves to 1 -spheres. Let us first explain this process with an example.

Example 5.8. Let $\varphi \equiv \exists y: y \rightarrow_{\mathcal{R}} y$. By Lemma 5.5, $\varphi^{S(r, x)}$ is equivalent to $\exists y$ : $x \rightarrow_{\mathcal{R}_{r}} y \rightarrow_{\mathcal{R}} y$. This property can be expressed as follows. There exists a rule $\left(S\left(x_{1}, \ldots, x_{n}\right), T\left(x_{1}, \ldots, x_{n}\right)\right) \in \mathcal{R}_{r}$ and there exist traces $u_{1} \in L\left(x_{1}\right), \ldots, u_{n} \in$ $L\left(x_{n}\right)$ such that $x=S\left(u_{1}, \ldots, u_{n}\right)$ and $T\left(u_{1}, \ldots, u_{n}\right) \rightarrow_{\mathcal{R}} T\left(u_{1}, \ldots, u_{n}\right)$. The existential quantification over all rules from $\mathcal{R}_{r}$ can be replaced by a finite disjunction since $\mathcal{R}_{r}$ is finite. Moreover, by Lemma 5.6, the statement $T\left(u_{1}, \ldots, u_{n}\right) \rightarrow_{\mathcal{R}}$ $T\left(u_{1}, \ldots, u_{n}\right)$ is equivalent to a Boolean combination $\theta$ of recognizable constraints for the factors $u_{i}$. Now the internalization of the resulting formula is the following: there exists a rule $\left(S\left(x_{1}, \ldots, x_{n}\right), T\left(x_{1}, \ldots, x_{n}\right)\right) \in \mathcal{R}_{r}$ and there exist convex subsets $X_{1}, \ldots, X_{n} \subseteq V$ (where $(V, E, \lambda)$ is the dependence graph of the central trace $x$ ) such that $\operatorname{fact}_{S}\left(V, X_{1}, \ldots, X_{n}\right)$ and $\Theta$ holds. Here $\Theta$ results from the Boolean combination $\theta$ by replacing every recognizable constraint $u_{i} \in L$ by an MSO-formula which expresses the fact that the restriction of the dependence graph $(V, E, \lambda)$ to the convex set $X_{i}$ satisfies some MSO-sentence defining $L \in \operatorname{REC}(\mathbb{M})$ which is possible by Theorem 5.7. 
For our further considerations we may w.l.o.g. assume that for some number $n$ every rule in the scattered rewriting system $\mathcal{R}_{r}$ is defined over exactly $n$ variables. This may be assumed since we always can add dummy variables $z$ with $L(z)=\{1\}$ to the end of the left- and right-hand side of a rule.

In the following theorem we have to include additional free variables $y_{1}, \ldots, y_{\ell}$ in order to be able to treat an existential quantifier inductively.

Theorem 5.9. Let $\varphi\left(x, y_{1}, \ldots, y_{\ell}\right)$ be an external first-order formula with free variables among $\left\{x, y_{1}, \ldots, y_{\ell}\right\}$ such that for any subformula of the form $\exists z \psi$, the formula $\psi$ has the form $x \rightarrow_{\mathcal{R}_{r}} z \wedge \psi^{\prime}$ for some formula $\psi^{\prime}{ }^{5}$ Further let $\left(S_{k}, T_{k}\right)_{1 \leq k \leq \ell}$ be a tuple of rules from $\mathcal{R}_{r}$. Then there exists an internal MSO-formula

$$
\operatorname{int}(\varphi)\left[\left(S_{k}, T_{k}\right)_{1 \leq k \leq \ell}\right]
$$

with free second-order variables among $\left\{Y_{k}^{i} \mid 1 \leq k \leq \ell, 1 \leq i \leq n\right\}$ such that for all dependence graphs $D_{t}=(V, E, \lambda)$ and sets $Y_{k}^{i} \subseteq V(1 \leq k \leq \ell, 1 \leq i \leq n)$ with $D_{t}=$ fact $_{S_{k}}\left(V, Y_{k}^{1}, Y_{k}^{2}, \ldots, Y_{k}^{n}\right)$, the following are equivalent:

- $D_{t} \models \operatorname{int}(\varphi)\left[\left(S_{k}, T_{k}\right)_{1 \leq k \leq \ell}\right]$

- $\mathcal{M}_{\mathcal{R}_{r}}^{\mathrm{red}}=\varphi\left(t, u_{1}, u_{2}, \ldots, u_{\ell}\right)$ where $u_{k}=T_{k}\left(D_{t} \uparrow_{Y_{k}^{1}}, D_{t} \uparrow_{Y_{k}^{2}}, \ldots, D_{t} \uparrow_{Y_{k}^{n}}\right)$.

Note that in the theorem above, we assume that $Y_{k}^{i}$ is a convex subset of $V$ since $D_{t}=$ fact $_{S_{k}}\left(V, Y_{k}^{1}, Y_{k}^{2}, \ldots, Y_{k}^{n}\right)$. Note also that $t \rightarrow_{\left(S_{k}, T_{k}\right)} u_{k}$ in the theorem above.

We start the proof of Theorem 5.9 with a few preprocessing steps:

1. First we may replace $\varphi\left(x, y_{1}, \ldots, y_{\ell}\right)$ by $\varphi\left(x, y_{1}, \ldots, y_{\ell}\right) \wedge \wedge_{1 \leq i \leq \ell} x \rightarrow_{\mathcal{R}_{r}} y_{i}$, because in Theorem 5.9 the free variables $y_{i}$ will be only interpreted by direct $\rightarrow_{\mathcal{R}_{r}}$-successors of the central trace $x$.

2. For $L \in \operatorname{REC}(\mathbb{M})$ let us define the scattered rewrite rule $\rho_{L}=\left(z_{L}, z_{L}\right)$ with $L\left(z_{L}\right)=L$. Then we may replace any atomic subformula of the form $y \in L$ by $y \rightarrow_{\rho_{L}} y$ (formally we also have to add $\rho_{L}$ to $\mathcal{R}_{r}$ ).

3. Finally, any atomic subformula of the form $y \rightarrow_{\rho} y$ can be replaced by the formula $\exists z\left(x \rightarrow_{\mathcal{R}_{r}} z \wedge y \rightarrow_{\rho} z \wedge y \rightarrow_{\rho_{\mathbb{M}}} z\right)$ where $z$ is a new variable (note that $y \rightarrow_{\rho_{\mathbb{M}}} z$ if and only if $y=z$ ). Here we can restrict the new variable $z$ to $\rightarrow_{\mathcal{R}_{r}}$-successors of the central trace $x$, because by the first step, every free variable $y_{i}$ has to satisfy $x \rightarrow_{\mathcal{R}_{r}} y_{i}$, and also every quantification in $\varphi$ is restricted to direct $\rightarrow_{\mathcal{R}_{r}}$-successors of $x$.

Thus, in the following we will only deal with subformulas of the form $y \rightarrow_{\rho} z$ where the variables $y$ and $z$ are distinct, with Boolean connectives, and with existential quantifications over $\rightarrow_{\mathcal{R}_{r}}$-successors of $x$. These considerations can be found in the following three claims that collectively prove Theorem 5.9.

Claim 1. Theorem 5.9 holds for the formula $\varphi \equiv\left(y_{1} \rightarrow_{\rho} y_{2}\right)$ where $\rho \in \mathbb{S}$ is a scattered rewrite rule.

\footnotetext{
${ }^{5}$ W.l.o.g. we may assume that $\varphi$ does not contain universal quantifiers.
} 
Proof. Fix two rules $\left(S_{k}, T_{k}\right) \in \mathcal{R}_{r}(k=1,2)$ and let $\left(S_{k}, T_{k}\right)$ be defined over the variables $y_{k}^{1}, \ldots, y_{k}^{n}$. Let $S_{k}=y_{k}^{1} a_{k}^{1} y_{k}^{2} a_{k}^{2} \cdots y_{k}^{n}$. For the construction of

$$
\operatorname{int}\left(y_{1} \rightarrow_{\rho} y_{2}\right)\left[\left(S_{1}, T_{1}\right),\left(S_{2}, T_{2}\right)\right]
$$

we will use all patterns $U_{i}=z_{i, 1} d_{i, 1} z_{i, 2} d_{i, 2} \cdots z_{i, n}$ and $V_{i}=z_{1, i} e_{1, i} z_{2, i} e_{2, i} \cdots z_{n, i}(1 \leq$ $i \leq n$ ) such that

$$
\begin{aligned}
& d_{i, j} \text { is a factor of } a_{2}^{j}, e_{i, j} \text { is a factor of } a_{1}^{i}, L\left(z_{i, j}\right)=\operatorname{alph}\left(z_{i, j}\right)^{*}, \text { and } \\
& \left\{\left(z_{i, j}, z_{p, q}\right) \mid(i<p \text { and } j>q) \text { or }\left(y_{1}^{i}, y_{1}^{p}\right) \in J \text { or }\left(y_{2}^{j}, y_{2}^{q}\right) \in J\right\} \subseteq J .
\end{aligned}
$$

Because of the restriction of these patterns to length $n$ and since all the traces mentioned in $U_{i}$ and $V_{i}$ are factors of some $a_{k}^{j}$, there are only finitely many patterns satisfying (8) up to renaming of variables.

Let $t \in \mathbb{M}$ with $D_{t}=(V, E, \lambda)$, and let $Y_{k}^{i} \subseteq V(1 \leq i \leq n, k=1,2)$ such that

$$
D_{t} \models \bigwedge_{k=1,2} \operatorname{fact}_{S_{k}}\left(V, Y_{k}^{1}, Y_{k}^{2}, \ldots, Y_{k}^{n}\right)
$$

Let $u_{k}=T_{k}\left(D_{t} \uparrow_{Y_{k}^{1}}, D_{t} \uparrow_{Y_{k}^{2}}, \ldots, D_{t} \uparrow_{Y_{k}^{n}}\right)$. We have to translate the statement $u_{1} \rightarrow_{\rho} u_{2}$ into an equivalent MSO-property of the sets $Y_{k}^{i}(1 \leq i \leq n, k=1,2)$.

Claim 1.1. There exist patterns $U_{i}$ and $V_{i}(1 \leq i \leq n)$ satisfying (8) and there exist sets $Z_{i, j} \subseteq V$ such that

$$
D_{t} \models \bigwedge_{1 \leq i \leq n} \operatorname{fact}_{U_{i}}\left(Y_{1}^{i}, Z_{i, 1}, Z_{i, 2}, \ldots, Z_{i, n}\right) \wedge \bigwedge_{1 \leq i \leq n} \operatorname{fact}_{V_{i}}\left(Y_{2}^{i}, Z_{1, i}, Z_{2, i}, \ldots, Z_{n, i}\right) .
$$

In order to prove Claim 1.1, note that (9) implies that there exist convex sets $A_{k}^{i} \subseteq V$ such that $D_{t} \uparrow_{A_{k}^{i}}$ represents the trace $a_{k}^{i}$ for $1 \leq i \leq n, k=1,2$, and the sequence $Y_{k}^{1}, A_{k}^{1}, Y_{k}^{2}, A_{k}^{2}, \ldots, Y_{k}^{n}$ is a factorization of $D_{t}$ for $k=1,2$. Define

$$
\begin{aligned}
C_{i, j} & =A_{1}^{i} \cap A_{2}^{j} \text { for } 1 \leq i, j<n, \\
D_{i, j} & =Y_{1}^{i} \cap A_{2}^{j} \text { for } 1 \leq i \leq n, 1 \leq j<n, \\
E_{i, j} & =A_{1}^{i} \cap Y_{2}^{j} \text { for } 1 \leq i<n, 1 \leq j \leq n, \\
Z_{i, j} & =Y_{1}^{i} \cap Y_{2}^{j} \text { for } 1 \leq i, j \leq n .
\end{aligned}
$$

The situation can be visualized by the following diagram. The rows represent the factors $Y_{1}^{i}$ and $A_{1}^{i}$ and the columns the factors $Y_{2}^{i}$ and $A_{2}^{i}$.

\begin{tabular}{c||c|c|c|c|c|c|}
\hline$Y_{1}^{n}$ & $Z_{n 1}$ & $D_{n 1}$ & $Z_{n, 2}$ & $D_{n, 2}$ & $\ldots$ & $Z_{n, n}$ \\
\hline$\vdots$ & $\vdots$ & $\vdots$ & $\vdots$ & $\vdots$ & $\vdots$ & $\vdots$ \\
\hline$A_{1}^{2}$ & $E_{2,1}$ & $C_{2,1}$ & $E_{2,2}$ & $C_{2,2}$ & $\ldots$ & $E_{2, n}$ \\
\hline$Y_{1}^{2}$ & $Z_{2,1}$ & $D_{2,1}$ & $Z_{2,2}$ & $D_{2,2}$ & $\ldots$ & $Z_{2, n}$ \\
\hline$A_{1}^{1}$ & $E_{1,1}$ & $C_{1,1}$ & $E_{1,2}$ & $C_{1,2}$ & $\ldots$ & $E_{1, n}$ \\
\hline$Y_{1}^{1}$ & $Z_{1,1}$ & $D_{1,1}$ & $Z_{1,2}$ & $D_{1,2}$ & $\ldots$ & $Z_{1, n}$ \\
\hline \hline & $Y_{2}^{1}$ & $A_{2}^{1}$ & $Y_{2}^{2}$ & $A_{2}^{2}$ & $\ldots$ & $Y_{2}^{n}$
\end{tabular}


The sequence $Z_{i, 1}, D_{i, 1}, Z_{i, 2}, D_{i, 2}, \ldots, Z_{i, n}\left(\right.$ resp. $\left.Z_{1, i}, E_{1, i}, Z_{2, i}, E_{2, i}, \ldots, Z_{n, i}\right)$ is a factorization of $D_{t} \uparrow_{Y_{1}^{i}}\left(\right.$ resp. $\left.D_{t} \uparrow_{Y_{2}^{i}}\right)$.

For $1 \leq i, j \leq n$, let $z_{i, j}$ be an external first-order variable with alph $\left(z_{i, j}\right)=\lambda\left(Z_{i, j}\right)$ and $L\left(z_{i, j}\right)=\operatorname{alph}\left(z_{i, j}\right)^{*}$. Since $\lambda\left(Z_{i, j}\right) \subseteq \lambda\left(Y_{1}^{i}\right) \cap \lambda\left(Y_{2}^{j}\right) \subseteq \operatorname{alph}\left(y_{1}^{i}\right) \cap \operatorname{alph}\left(y_{2}^{j}\right)$ and $\lambda\left(Z_{i, j}\right) \times \lambda\left(Z_{p, q}\right) \subseteq I$ whenever $i<p$ and $j>q$ (see Lemma 3.1), we obtain

$$
\left\{\left(z_{i, j}, z_{p, q}\right) \mid(i<p \text { and } j>q) \text { or }\left(y_{1}^{i}, y_{1}^{p}\right) \in J \text { or }\left(y_{2}^{j}, y_{2}^{q}\right) \in J\right\} \subseteq J .
$$

Furthermore let $\left.D_{t}\right|_{D_{i, j}}\left(\right.$ resp $\left.D_{t}\right|_{E_{i, j}}$ ) represent the trace $d_{i, j} \in \mathbb{M}$ (resp. $e_{i, j} \in \mathbb{M}$ ). Then the patterns

$$
U_{i}=z_{i, 1} d_{i, 1} z_{i, 2} d_{i, 2} \cdots z_{i, n} \text { and } V_{i}=z_{1, i} e_{1, i} z_{2, i} e_{2, i} \cdots z_{n, i}
$$

for $1 \leq i \leq n$ satisfy (8). Finally from the diagram above we can easily deduce (10). This proves Claim 1.1.

Let us fix patterns $U_{i}, V_{i}(1 \leq i \leq n)$ satisfying (8) and sets $Z_{i, j} \subseteq V$ satisfying (10). Thus, $\left.\left.D_{t} \uparrow_{Y_{1}^{i}}=U_{i}\left(D_{t} \uparrow_{Z_{i, 1}}, D_{t}\right\rceil_{Z_{i, 2}}, \ldots, D_{t}\right\rceil_{Z_{i, n}}\right)$, which has to be read as an identity between traces. Let the pattern $T_{1}\left[y_{1}^{i} / U_{i}\right]$ (resp. $T_{2}\left[y_{2}^{i} / V_{i}\right]$ ) result from $T_{1}$ (resp. $T_{2}$ ) by substituting for all $1 \leq i \leq n$ the pattern $U_{i}$ (resp. $V_{i}$ ) for the variable $y_{1}^{i}$ (resp. $\left.y_{2}^{i}\right)$. With $u_{1}=T_{1}\left(D_{t} \uparrow_{Y_{1}^{1}}, D_{t} \uparrow_{Y_{1}^{2}}, \ldots, D_{t} \uparrow_{Y_{1}^{n}}\right)$, we obtain $u_{1}=$ $T_{1}\left[y_{1}^{i} / U_{i}\right]\left(D_{t}\left\lceil_{Z_{1,1}}, \ldots, D_{t}\left\lceil_{Z_{n, n}}\right)\right.\right.$. The equation $u_{2}=T_{2}\left[y_{2}^{i} / V_{i}\right]\left(D_{t}\left\lceil_{Z_{1,1}}, \ldots, D_{t}\left\lceil_{Z_{n, n}}\right)\right.\right.$ follows similarly. Since we can take a finite disjunction over all patterns $U_{i}, V_{i}(1 \leq$ $i \leq n$ ) satisfying (8) followed by an existential quantification over all sets $Z_{i, j} \subseteq V$ satisfying (10), it suffices to translate the statement

$$
T_{1}\left[y_{1}^{i} / U_{i}\right]\left(D _ { t } \left\lceilZ_{1,1}, \ldots, D_{t}\left\lceil Z_{n, n}\right) \rightarrow_{\rho} T_{2}\left[y_{2}^{i} / V_{i}\right]\left(D _ { t } \left\lceilZ_{1,1}, \ldots, D_{t}\left\lceil Z_{n, n}\right)\right.\right.\right.\right.
$$

into an equivalent MSO-property. The definition of the patterns $T_{1}\left[y_{1}^{i} / U_{i}\right], T_{2}\left[y_{2}^{i} / V_{i}\right]$ and (8) imply that type $\left(T_{1}\left[y_{1}^{i} / U_{i}\right]\right) \equiv_{J}$ type $\left(T_{2}\left[y_{2}^{i} / V_{i}\right]\right){ }^{6}$ From (10) we get $D_{t}\left\lceil\sum_{i, j} \in\right.$ $L\left(z_{i, j}\right)$. Thus we can apply Lemma 5.6, which tells us that (11) is equivalent to a Boolean combination of formulae of the form $D_{t} \uparrow_{Z_{i, j}} \in L$ for some $L \in \operatorname{REC}(\mathbb{M})$. By Theorem 5.7, this Boolean combination of external first-order formulae $D_{t} \uparrow_{z_{i, j}} \in L$ can be translated into an equivalent Boolean combination of internal MSO-formulae $\psi\left\lceil_{Z_{i, j}}\right.$, where $\psi\left\lceil_{Z_{i, j}}\right.$ results from an MSO-sentence $\psi$ defining $L$ by restricting all quantifications in $\psi$ to $Z_{i, j}$.

Claim 2. Theorem 5.9 holds for the formulas $\varphi \equiv\left(y_{1} \rightarrow_{\rho} x\right)$ and $\varphi \equiv\left(x \rightarrow_{\rho} y_{1}\right)$.

Proof. Let int $\left(y_{1} \rightarrow_{\rho} x\right)\left[\left(S_{1}, T_{1}\right)\right]$ be

$$
\exists \bar{Y}_{2}\left(\operatorname{fact}_{S_{2}}\left(V, \bar{Y}_{2}\right) \wedge \operatorname{int}\left(y_{1} \rightarrow_{\rho} y_{2}\right)\left[\left(S_{1}, T_{1}\right),\left(S_{2}, T_{2}\right)\right]\right)
$$

where $\bar{Y}_{2}$ is the sequence of variables $Y_{2}^{1}, \ldots, Y_{2}^{n}$ and $\left(S_{2}, T_{2}\right)=\rho_{\mathbb{M}}$. The formula $\operatorname{int}\left(x \rightarrow_{\rho} y_{1}\right)\left[\left(S_{1}, T_{1}\right)\right]$ can be defined analogously. This gives a reduction to Claim 1.

\footnotetext{
${ }^{6}$ This can be shown completely analogously to the equality type $(P) \equiv_{J} \operatorname{type}(R)$ in the proof of Lemma 5.4.
} 
Claim 3. If Theorem 5.9 holds for the formulae $\varphi$ and $\psi$, then it holds for the formulae $\varphi \wedge \psi, \neg \varphi$ and $\exists y\left(x \rightarrow_{\mathcal{R}_{r}} y \wedge \varphi\right)$.

Proof. Conjunction and negation are easy:

$$
\begin{aligned}
\operatorname{int}(\varphi \wedge \psi)\left[\left(S_{k}, T_{k}\right)_{1 \leq k \leq \ell}\right] & \equiv \operatorname{int}(\varphi)\left[\left(S_{k}, T_{k}\right)_{1 \leq k \leq \ell}\right] \wedge \operatorname{int}(\psi)\left[\left(S_{k}, T_{k}\right)_{1 \leq k \leq \ell}\right] \\
\operatorname{int}(\neg \varphi)\left[\left(S_{k}, T_{k}\right)_{1 \leq k \leq \ell}\right] & \equiv \neg\left(\operatorname{int}(\varphi)\left[\left(S_{k}, T_{k}\right)_{1 \leq k \leq \ell}\right]\right) .
\end{aligned}
$$

In order to define $\operatorname{int}\left(\exists y\left(x \rightarrow_{\mathcal{R}_{r}} y \wedge \varphi\right)\left[\left(S_{k}, T_{k}\right)_{1 \leq k<\ell}\right]\right)$, let $\varphi\left(x, y_{1}, \ldots, y_{\ell}\right)$ be an external first-order formula whose free variables are contained in $\left\{x, y_{1}, \ldots, y_{\ell}\right\}$ and let w.l.o.g. $y=y_{\ell}$. Let $\overline{Y_{\ell}}$ be the sequence of variables $Y_{\ell}^{1}, \ldots, Y_{\ell}^{n}$ and set

$$
\begin{aligned}
& \operatorname{int}\left(\exists y _ { \ell } \left(x \rightarrow \mathcal{R}_{r}\right.\right.\left.\left.y_{\ell} \wedge \varphi\right)\right)\left[\left(S_{k}, T_{k}\right)_{1 \leq k<\ell}\right] \equiv \\
& \bigvee_{\left(S_{\ell}, T_{\ell}\right) \in \mathcal{R}_{r}} \exists \bar{Y}_{\ell}\left(\operatorname{fact}_{S_{\ell}}\left(V, \bar{Y}_{\ell}\right) \wedge \operatorname{int}(\varphi)\left[\left(S_{k}, T_{k}\right)_{1 \leq k \leq \ell}\right]\right) .
\end{aligned}
$$

Suppose $Y_{k}^{i} \subseteq V(1 \leq k<\ell, 1 \leq i \leq n)$ are such that

$$
D_{t} \models \operatorname{fact}_{S_{k}}\left(V, Y_{k}^{1}, Y_{k}^{2}, \ldots, Y_{k}^{n}\right) .
$$

If $D_{t}=(V, E, \lambda) \models \operatorname{int}\left(\exists y_{\ell}\left(x \rightarrow_{\mathcal{R}_{r}} y_{\ell} \wedge \varphi\right)\right)\left[\left(S_{k}, T_{k}\right)_{1 \leq k<\ell}\right]$ then there is a scattered rewrite rule $\left(S_{\ell}, T_{\ell}\right) \in \mathcal{R}_{r}$ and there are sets $Y_{\ell}^{i} \subseteq V(1 \leq i \leq n)$ such that $D_{t} \models \operatorname{fact}_{S_{\ell}}\left(V, \bar{Y}_{\ell}\right)$ and $D_{t} \models \operatorname{int}(\varphi)\left[\left(S_{k}, T_{k}\right)_{1 \leq k \leq \ell}\right]$. Inductively we obtain $\mathcal{M}_{\mathcal{R}_{r}}^{\mathrm{red}} \models \varphi\left(t, u_{1}, u_{2}, \ldots, u_{\ell}\right)$ where $u_{k}=T_{k}\left(D_{t} \uparrow_{Y_{k}^{1}}, D_{t} \uparrow_{Y_{k}^{2}}, \ldots, D_{t} \uparrow_{Y_{k}^{n}}\right)$ for $1 \leq k \leq \ell$. Since $t \rightarrow{ }_{\left(S_{\ell}, T_{\ell}\right)} u_{\ell}$, it follows $\mathcal{M}_{\mathcal{R}_{r}}^{\text {red }}=\left(\exists y_{\ell}\left(x \rightarrow_{\mathcal{R}_{r}} y_{\ell} \wedge \varphi\right)\right)\left(t, u_{1}, u_{2}, \ldots, u_{\ell-1}\right)$.

On the other hand if $\mathcal{M}_{\mathcal{R}_{r}}^{\text {red }} \models\left(\exists y_{\ell}\left(x \rightarrow_{\mathcal{R}_{r}} y_{\ell} \wedge \varphi\right)\right)\left(t, u_{1}, u_{2}, \ldots, u_{\ell-1}\right)$ for the traces $u_{k}=T_{k}\left(D_{t} \uparrow_{Y_{k}^{1}}, D_{t} \uparrow_{Y_{k}^{2}}, \ldots, D_{t} \uparrow_{Y_{k}^{n}}\right)(1 \leq k<\ell)$, then there is a rule $\left(S_{\ell}, T_{\ell}\right) \in \mathcal{R}_{r}$ and there are sets $Y_{\ell}^{i} \subseteq V(1 \leq i \leq n)$ such that $D_{t}=$ fact $_{S_{\ell}}\left(V, \bar{Y}_{\ell}\right)$ and $\mathcal{M}_{\mathcal{R}_{r}}^{\text {red }} \models \varphi\left(t, u_{1}, u_{2}, \ldots, u_{\ell}\right)$, where $u_{\ell}=T_{\ell}\left(D_{t} \uparrow_{Y_{\ell}^{1}}, D_{t} \uparrow_{Y_{\ell}^{2}}, \ldots, D_{t} \uparrow_{Y_{\ell}^{n}}\right)$. Hence by induction, the dependence graph $D_{t}$ satisfies the formula int $(\varphi)\left[\left(S_{k}, T_{k}\right)_{1 \leq k \leq \ell}\right]$, which finally implies $D_{t} \models \operatorname{int}\left(\exists y_{\ell}\left(x \rightarrow_{\mathcal{R}_{r}} y_{\ell} \wedge \varphi\right)\right)\left[\left(S_{k}, T_{k}\right)_{1 \leq k<\ell}\right]$.

This finishes the inductive proof of Theorem 5.9.

Remark 5.10. In the proof of Theorem 5.9 we have translated an external first-order property into an internal MSO property of the underlying dependence graph. A closer analysis shows that first-order logic over dependence graphs is in fact sufficient for the process of internalization. The reason is that we only used second-order quantifications over convex subsets. But a convex subset can be represented by the tuple of its minimal and maximal vertices. This tuple consists of at most $2 \cdot|\Sigma|$ vertices. Moreover, all recognizable sets that we have constructed in Section 4 are easily seen to be first-order definable. 


\subsection{Proof of Theorem 3.8}

Theorem 5.11. Let $r \geq 0$ and let $\varphi(x)$ be a first-order formula over the signature of $\mathcal{M}_{\mathcal{R}}^{\text {red }}$ that contains at most $x$ free. Then the set $\left\{t \in \mathbb{M} \mid \mathcal{M}_{\mathcal{R}}^{\text {red }} \models \varphi(x)^{S(r, x)}(t)\right\}$ is a recognizable subset of $\mathbb{M}$.

Proof. Let $\mathcal{R}_{r}$ be the scattered rewriting system from Lemma 5.5 and let $t \in \mathbb{M}$ be some trace. By Lemma 5.5, $\mathcal{M}_{\mathcal{R}}^{\text {red }} \models \varphi(x)^{S(r, x)}(t)$ if and only if $\mathcal{M}_{\mathcal{R}_{r}}^{\text {red }} \models \varphi(x)^{S(1, x)}(t) .^{7}$ Note that the formula $\varphi(x)^{S(1, x)}$ satisfies the conditions of Theorem 5.9 (if we write $x \rightarrow_{\mathcal{R}_{r}} z$ instead of $\left.d(x, z) \leq 1\right)$ where, in addition, the tuple $\left(S_{k}, T_{k}\right)_{1 \leq k \leq \ell}$, the sets $Y_{k}^{i} \subseteq V$, and the traces $u_{k}$ disappear. In particular, the MSO-formula int $\left(\varphi(x)^{S(1, x)}\right)$ does not contain any free variables, i.e., it is an MSO-sentence. Thus, we obtain from the external first-order formula $\varphi(x)$ the internal MSO-sentence int $\left(\varphi(x)^{S(1, x)}\right)$ such that $D_{t} \models \operatorname{int}\left(\varphi(x)^{S(1, x)}\right)$ if and only if $\mathcal{M}_{\mathcal{R}}^{\text {red }} \models \varphi(x)^{S(r, x)}(t)$. Hence the set $\left\{t \in \mathbb{M} \mid \mathcal{M}_{\mathcal{R}}^{\text {red }}=\varphi(x)^{S(r, x)}(t)\right\}$ is defined by the MSO-sentence $\operatorname{int}\left(\varphi(x)^{S(1, x)}\right)$; it is therefore recognizable by Theorem 5.7.

We finish this section with the proof of our main result, Theorem 3.8.

Proof of Theorem 3.8. By Gaifman's Theorem (Theorem 5.1) and the discussion following that theorem, it suffices to decide whether a sentence of the form

$$
\exists x_{1} \cdots \exists x_{m}\left\{\bigwedge_{1 \leq i<j \leq m} d\left(x_{i}, x_{j}\right)>2 r \wedge \bigwedge_{1 \leq i \leq m} \psi\left(x_{i}\right)^{S\left(r, x_{i}\right)}\right\}
$$

holds in $\mathcal{M}_{\mathcal{R}}^{\text {red }}$. Here $\psi\left(x_{i}\right)$ is a first-order formula over the signature of $\mathcal{M}_{\mathcal{R}}^{\text {red }}$ that results from a formula $\psi(x)$ by replacing every free occurrence of $x$ by $x_{i}$. By Theorem 5.11, the set $L=\left\{t \in \mathbb{M} \mid \mathcal{M}_{\mathcal{R}}^{\text {red }} \models \psi(x)^{S(r, x)}(t)\right\}$ is recognizable. Hence we can check whether $L$ is infinite. If this is the case then $L$ contains traces of arbitrary length. Since the application of a rewrite rule from the finite system $\mathcal{R}$ can change the length of a trace only by some fixed amount, there exist infinitely many traces $t_{i} \in L, i \in \mathbb{N}$, such that $d_{\mathcal{M}_{\mathcal{R}}^{\text {red }}}\left(t_{i}, t_{j}\right)>2 r$ for $i<j$. Hence (12) is true. On the other hand if $L$ is finite, then we can enumerate all elements of $L$ and calculate their $r$-spheres with respect to $\mathcal{R}$. In this way we can check whether there are at least $m$ traces $t_{1}, \ldots, t_{m} \in L$ such that $d_{\mathcal{M}_{\mathcal{R}}^{\text {red }}}\left(t_{i}, t_{j}\right)>2 r$ for $i<j$. Hence the decidability follows.

\subsection{An extension of first-order logic}

First-order logic can be extended by modulo counting quantifiers [59]; the resulting logic is called FO+MOD. Since there is no locality theorem known for this logic, ${ }^{8}$ our decidability proof for the first-order theory of $\mathcal{M}^{\text {red }}$ does not work for this more expressive logic; but the second step of our proof, i.e., the recognizability of the set of

\footnotetext{
${ }^{7}$ Note that $\varphi(x)^{S(r, x)}$ is built using the signature of $\mathcal{M}_{\mathcal{R}}^{\text {red }}$ while $\varphi(x)^{S(1, x)}$ uses the larger signature of $\mathcal{M}_{\mathcal{R}_{r}}^{\text {red }}$.

${ }^{8}$ Libkin [34-36] and Nurmonen [53] proved locality theorems for counting logics including modulo counting, but not in the form of Theorem 5.1. We could not make them work in our situation.
} 
traces satisfying some local formula in FO (cf. Theorems 5.9 and 5.7) extends to the logic FO+MOD. Thus, we obtain the decidability of local properties expressed in the logic FO+MOD.

The only difference between FO+MOD and FO is that we now have a second type of quantifiers: if $\varphi$ is a formula of FO+MOD, then $\exists^{(p, q)} x \varphi$ is a formula as well for $p, q \in \mathbb{N}$ and $p<q$. Then $\mathcal{M}^{\text {red }} \models \exists^{(p, q)} x \varphi$ if the number of traces $t \in \mathbb{M}$ with $\mathcal{M}^{\text {red }} \models \varphi(t)$ is finite and congruent $p$ modulo $q$.

The logic MSO+MOD We will also extend the monadic second-order logic for dependence graphs (MSO) by modulo-counting facilities for both set and first-order variables. MSO+MOD is the extension of MSO by the quantifiers $\exists^{(p, q)}\left(X_{i}\right)_{i \leq n}$ for a tuple of first-order or second-order variables $X_{i}$ and $p, q \in \mathbb{N}$ with $p<q$. This quantifier for set variables is only allowed in conjunction with the formula $\operatorname{conv}(X)$ asserting that $X$ is convex: if $\varphi$ is a formula of MSO+MOD then also the formula $\exists^{(p, q)}\left(X_{i}\right)_{i \leq n}\left(\bigwedge_{i \leq n} \operatorname{conv}\left(X_{i}\right) \wedge \varphi\right)$ and $\exists^{(p, q)}\left(x_{i}\right)_{i \leq n} \varphi$ belong to MSO+MOD. The restriction to convex sets will be assumed implicitly in the following. A dependence graph $D_{t}=(V, E, \lambda)$ satisfies $\exists^{(p, q)}\left(X_{i}\right)_{i \leq n} \psi$ if the number of set tuples $\left(X_{i}\right)_{i \leq n}$ with $X_{i} \subseteq V$ such that $D_{t} \models \psi\left(\left(X_{i}\right)_{i \leq n}\right)$ is congruent $p$ modulo $q$. The interpretation of the quantifier $\exists^{(p, q)}$ applied to first-order variables is similar. Note that, differently from FO+MOD, we did not require explicitly the set of witnesses to be finite; this is not needed since any dependence graph is finite.

We show that any property expressible in MSO+MOD can also be expressed in monadic second-order logic. The reason for introducing this extension of MSO is that the analog of Theorem 5.9 is shown much simpler in terms of MSO+MOD.

Lemma 5.12. Let $\varphi$ be a sentence of MSO+MOD. Then there exists a sentence $\psi$ of MSO such that for any $t \in \mathbb{M}, D_{t}=\varphi$ if and only if $D_{t} \models \psi$.

Proof. We first eliminate modulo quantifications over tuples of sets by modulo quantifications over single sets: the formula $\exists^{(p, q)}\left(X_{i}\right)_{1 \leq i \leq n} \varphi$ holds in the dependence graph $D_{t}$ if and only if for some numbers $0 \leq p_{i}<q$ with $\sum_{0 \leq i<q}\left(i \cdot p_{i}\right) \equiv p \bmod q$, we have

$$
D_{t} \models \bigwedge_{0 \leq i<q} \exists^{\left(p_{i}, q\right)} X_{1} \exists^{(i, q)}\left(X_{j}\right)_{2 \leq j \leq n} \varphi
$$

Recall that the modular set quantification runs over convex subsets of the underlying dependence graph, only. These convex sets can be encoded by the tuples of their minimal and maximal elements. In order to count correctly, we have to rule out permutations in these antichains. This is achieved by a linear order $\sqsubseteq$ on the set $\Sigma$ of letters. Let $m$ be the number of letters in $\Sigma$. Then the convex set $X$ is replaced by the two $m$-tuples $\left(x_{i}^{1}\right)_{1 \leq i \leq m}$ and $\left(x_{i}^{2}\right)_{1 \leq i \leq m}$ of minimal and maximal elements such that, for $1 \leq i<m$, we have either $\lambda\left(x_{i}^{j}\right) \sqsubset \lambda\left(x_{i+1}^{j}\right)$ or $x_{i}^{j}=x_{i+1}^{j}=x_{i+2}^{j}=\cdots=x_{m}^{j}$ 
for $j=1,2$; this can be expressed by a first-order formula $\mathrm{NF}\left(\left(x_{i}\right)_{1 \leq i \leq m}\right)$. Thus, the formula $\exists^{(p, q)} X \psi$ becomes

$$
\exists^{(p, q)}\left(x_{i}^{j}\right)_{\substack{1 \leq i \leq m \\
1 \leq j \leq 2}}\left\{\begin{array}{l}
\psi^{\prime} \wedge\left(\left(x_{i}^{1}\right) \text { and }\left(x_{i}^{2}\right) \text { form antichains }\right) \wedge \\
\operatorname{NF}\left(\left(x_{i}^{1}\right)_{1 \leq i \leq m}\right) \wedge \mathrm{NF}\left(\left(x_{i}^{2}\right)_{1 \leq i \leq m}\right) \wedge \\
\bigwedge_{1 \leq i \leq m} \bigvee_{1 \leq k \leq m} x_{i}^{1} \preceq x_{k}^{2} \wedge \bigwedge_{1 \leq k \leq m} \bigvee_{1 \leq i \leq m} x_{i}^{1} \preceq x_{k}^{2}
\end{array}\right\}
$$

In this formula, $\psi^{\prime}$ is obtained from $\psi$ by replacing any subformula of the form $x \in X$ by

$$
\bigvee_{1 \leq i, k \leq m} x_{i}^{1} \preceq x \preceq x_{k}^{2} .
$$

This eliminates all modular quantifications over convex sets by modulo quantification over tuples of first-order variables. In a second step, these quantifications over tuples can be eliminated as described above for tuples of set variables. This results in a formula from MSO+MOD where modulo quantification is applied to single first-order variables, only. These modulo quantifications can be eliminated since any dependence graph over $\Sigma$ can be covered by $m=|\Sigma|$ chains: the formula $\exists^{(p, q)} x \varphi$ is equivalent to the formula

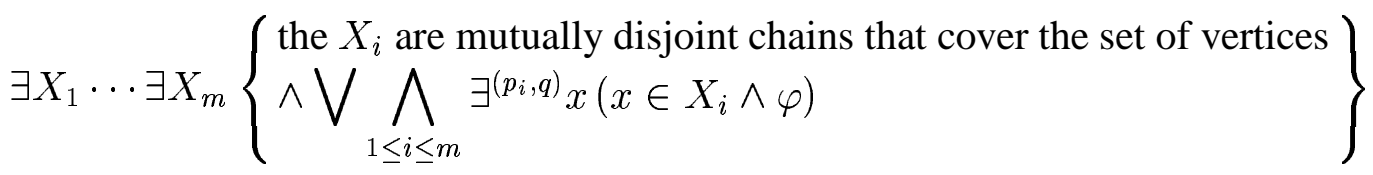

where the disjunction runs over all tuples $p_{1}, p_{2}, \ldots, p_{m}$ with $0 \leq p_{i}<q$ and $\sum p_{i} \equiv$ $p \bmod q$. This formula restricts the modulo quantifiers $\exists^{\left(p_{i}, q\right)} x$ to elements of the chain $X_{i}$ - these restricted modulo quantifications can be expressed in MSO as any introductory text on MSO for words explains (see, e.g., [61]).

From FO+MOD to MSO+MOD Next, we will prove an analogue of Theorem 5.9 for the logic FO+MOD. By Lemma 5.12, it suffices to reduce a local formula of FO+MOD to a formula of MSO+MOD. The statement of the following result is identical to Theorem 5.9 except that first-order logic (resp. MSO) is replaced by FO+MOD (resp. MSO+MOD).

Theorem 5.13. Let $\varphi\left(x, y_{1}, \ldots, y_{\ell}\right)$ be an external formula of FO+MOD with free variables among $\left\{x, y_{1}, \ldots, y_{\ell}\right\}$ such that for any subformula of the form $\exists z \psi$ or $\exists^{(p, q)} z \psi$, the formula $\psi$ is of the form $x \rightarrow_{\mathcal{R}_{r}} z \wedge \psi^{\prime}$ for some $\psi^{\prime}$. Further, let $\left(S_{k}, T_{k}\right)_{1 \leq k \leq \ell}$ be a tuple of rules from $\mathcal{R}_{r}$. Then there exists an internal formula of $\mathrm{MSO}+\mathrm{MOD}$

$$
\operatorname{int}(\varphi)\left[\left(S_{k}, T_{k}\right)_{1 \leq k \leq \ell}\right]
$$

with free second-order variables among $\left\{Y_{k}^{i} \mid 1 \leq k \leq \ell, 1 \leq i \leq n\right\}$ such that for all dependence graphs $D_{t}=(V, E, \lambda)$ and sets $Y_{k}^{i} \subseteq V(1 \leq k \leq \ell, 1 \leq i \leq n)$ with $D_{t}=$ fact $_{S_{k}}\left(V, Y_{k}^{1}, Y_{k}^{2}, \ldots, Y_{k}^{n}\right)$, the following are equivalent: 


$$
\begin{aligned}
& \text { - } D_{t} \models \operatorname{int}(\varphi)\left[\left(S_{k}, T_{k}\right)_{1 \leq k \leq \ell}\right] \\
& \text { - } \mathcal{M}_{\mathcal{R}_{r}}^{\text {red }}=\varphi\left(t, u_{1}, u_{2}, \ldots, u_{\ell}\right) \text { where } u_{k}=T_{k}\left(D_{t} \uparrow_{Y_{k}^{1}}, D_{t} \uparrow_{Y_{k}^{2}}, \ldots, D_{t} \uparrow_{Y_{k}^{n}}\right) .
\end{aligned}
$$

The proof proceeds along the lines of the proof of Theorem 5.9. Actually, the preprocessing steps and the proofs of Claims 1 to 3 go through verbatim and it only remains to consider formulae of the form $\exists^{(p, q)} y\left(x \rightarrow_{\mathcal{R}_{r}} y \wedge \varphi\right)$ :

Claim 4. If Theorem 5.13 holds for the formula $\varphi\left(x, y_{1}, \ldots, y_{\ell}\right)$, then it holds for the formula $\exists^{(p, q)} y_{\ell}\left(x \rightarrow_{\mathcal{R}_{r}} y_{\ell} \wedge \varphi\right)$.

Proof. One might be tempted to follow the proof of Claim 3 where we dealt with the usual quantifier. There, the existence of a trace $y_{\ell}$ corresponds to the existence of a tuple of sets $Y_{\ell}^{i}$ that factor the central trace $t$ in a certain way. The problem we have to face here is that there is no bijection between these tuples of convex sets and the elements of the sphere around $t$. Here, differently from Theorem 5.9, we need a bijection since we want to count the witnesses of a formula. Therefore, we now define a linear order on (tuples of) convex sets that is moreover MSO-definable:

Let $t \in \mathbb{M}$ and $D_{t}=(V, E, \lambda)$ be its dependence graph. Let $\preceq=E^{*}$. Fix an arbitrary linear order $\sqsubset$ on the alphabet $\Sigma$. We can lift $\sqsubset$ to a linear order on $2^{\Sigma}$ by defining $A \sqsubset B(A, B \subseteq \Sigma)$ if and only if

$$
\exists a \in B \backslash A \forall b \in \Sigma\{b \sqsubset a \Rightarrow(b \in A \Leftrightarrow b \in B)\} .
$$

Now assume that $X, Y \subseteq V$ are two different convex sets in the dependence graph $D_{t}$. We set $X \triangleleft Y$ if and only if

- $\lambda(X) \sqsubset \lambda(Y)$ or

- $\lambda(X)=\lambda(Y)$ and there is $a \in \Sigma$ such that for all $b \sqsubset a$ we have $X \cap \lambda^{-1}(b)=$ $Y \cap \lambda^{-1}(b)$ whereas either

- $\min _{\preceq}\left(X \cap \lambda^{-1}(a)\right) \prec \min _{\preceq}\left(Y \cap \lambda^{-1}(a)\right)$ or

- $\min _{\preceq}\left(X \cap \lambda^{-1}(a)\right)=\min _{\preceq}\left(Y \cap \lambda^{-1}(a)\right)$ and $\max _{\preceq}\left(X \cap \lambda^{-1}(a)\right) \prec \max _{\preceq}\left(Y \cap \lambda^{-1}(a)\right)$.

Then $\triangleleft$ is a linear order on convex subsets: if $X$ is convex and $a \in \Sigma$ then the set of all $a$-labeled nodes in $X$ is an interval within the chain of all $a$-labeled nodes in $D_{t}$. If $\lambda(X)=\lambda(Y)$ but $X \neq Y$ then there is a smallest $a \in \Sigma$ such that the intervals of $a$-labeled nodes in $X$ and $Y$, respectively, are different. We first compare the minimal nodes in these two intervals and then, if the minimal nodes are equal, the maximal nodes.

We denote the lexicographic extension of $\triangleleft$ to $n$-tuples of convex sets by $\triangleleft$ again. Then one can write down an internal MSO-formula less with free set variables $X_{1}, \ldots, X_{n}$ and $Y_{1}, \ldots, Y_{n}$ such that, for $t \in \mathbb{M}$ with $D_{t}=(V, E, \lambda)$ and $X_{i}, Y_{i} \subseteq V$ convex, we have

$$
D_{t} \models \operatorname{less}(\bar{X}, \bar{Y}) \text { if and only if } \bar{X} \triangleleft \bar{Y} \text {. }
$$


Recall that a rule $(S, T) \in \mathcal{R}_{r}$ and a tuple $\bar{Y}$ with $D_{t}=$ fact $_{S}(V, \bar{Y})$ represent a $\rightarrow_{\mathcal{R}_{r}}$ successor of the central trace $t$. Let $\sqsubset$ be an arbitrary linear order on $\mathcal{R}_{r}$. We can write an MSO-formula least ${ }_{(S, T)}(\bar{Y})$ stating that $(\bar{Y}, S, T)$ is the least representative of some $\rightarrow_{\mathcal{R}_{r}}$-successor of $t{ }^{9}$

$$
\begin{gathered}
\bigwedge_{\substack{(U, V) \in \mathcal{R}_{r} \\
(U, V) \sqsubset(S, T)}} \forall \bar{Z}\left\{\operatorname{fact}_{U}(V, \bar{Z}) \Rightarrow \operatorname{int}(y=z)[(S, T),(U, V)]\right\} \wedge \\
\forall \bar{Z}\left\{\left(\operatorname{fact}_{S}(V, \bar{Z}) \wedge \operatorname{int}(y=z)[(S, T),(S, T)]\right) \Rightarrow \operatorname{less}(\bar{Y}, \bar{Z})\right\}
\end{gathered}
$$

Now the internal equivalent $\operatorname{int}\left(\exists^{(p, q)} y_{\ell}\left(x \rightarrow_{\mathcal{R}_{r}} y_{\ell} \wedge \varphi\right)\right)\left[\left(S_{k}, T_{k}\right)_{1 \leq k<\ell}\right]$ of the external FO+MOD formula $\exists^{(p, q)} y_{\ell}\left(x \rightarrow_{\mathcal{R}_{r}} y_{\ell} \wedge \varphi\right)$ is the disjunction over all tuples $\left(n_{\rho}\right)_{\rho \in \mathcal{R}_{r}}$ with $\sum_{\rho \in \mathcal{R}_{r}} n_{\rho} \equiv p \bmod q$ of the following formula:

$$
\bigwedge_{\rho=\left(S_{\ell}, T_{\ell}\right) \in \mathcal{R}_{r}}\left(\exists^{\left(n_{\rho}, q\right)} \bar{Y}\left(\operatorname{fact}_{S_{\ell}}(V, \bar{Y}) \wedge \operatorname{least}_{\left(S_{\ell}, T_{\ell}\right)}(\bar{Y}) \wedge \operatorname{int}(\varphi)\left[\left(S_{k}, T_{k}\right)_{1 \leq k \leq \ell}\right]\right)\right)
$$

The remaining reasoning is similar to that in Claim 3.

After having shown Claim 4, Theorem 5.13 follows by induction similarly to Theorem 5.9.

Similarly to the main result Theorem 3.8, we can now show that the FO+MODtheory of $r$-spheres is decidable:

Theorem 5.14. There is an algorithm that, on input of an independence alphabet $(\Sigma, I)$, a natural number $r \geq 0$, and a formula $\varphi(x)$ of FO+MOD over the signature of the structure $\mathcal{M}^{\mathrm{red}}$, decides whether there exists $t \in \mathbb{M}$ with $\mathcal{M}^{\mathrm{red}} \models \varphi(x)^{S(r, x)}(t)$.

Proof. By Theorem 5.13 and Lemma 5.12, the set

$$
L=\left\{t \in \mathbb{M} \mid \mathcal{M}_{\mathcal{R}}^{\mathrm{red}} \models \varphi(x)^{S(r, x)}(t)\right\}
$$

can be characterized by a sentence of monadic second-order logic. Hence it is recognizable by Theorem 5.7. Since the emptiness of recognizable languages is decidable, the result follows.

\section{Complexity issues}

Recall that the first step in our decidability proof for Theorem 3.8 was an application of Gaifman's Theorem. To the knowledge of the authors all known translations of a first-order sentence into a Boolean combination of local sentences are not elementary in the worst case, thus our decision procedure is far from efficient. In this section, we show that one cannot avoid this nonelementary complexity.

\footnotetext{
${ }^{9}$ We assume that the MSO-formula int $(y=z)$ has free set variables $\bar{Y}, \bar{Z}$, where the tuple $\bar{Y}$ (resp. $\bar{Z}$ ) corresponds to $y$ (resp. $z$ ).
} 
To this aim, we construct an independence alphabet $(\Sigma, I)$ and a trace rewriting system $\mathcal{R}$ over $\mathbb{M}$ such that the first-order theory of the graph $\left(\mathbb{M}, \rightarrow_{\mathcal{R}}\right)$ is not elementary. Thus, our use of Gaifman's translation does not lead to an unreasonable inefficiency. We actually show a slightly stronger result, namely that the set of valid local sentences for a fixed radius is not elementary. In other words, the complexity of the decision question is already present when restricting to local sentences.

We prove the lower bound by reducing the first-order theory of finite labeled linear orders. In order to formulate this, we take the MSO-logic over dependence graphs from Section 5.3 but forbid the use of second-order variables. The resulting formulae are called first-order formulae over dependence graphs. For our further considerations we will use this logic only for dependence graphs $D_{t}$ where $t$ is in fact a word $t \in \Gamma^{*}$. In this case the relation symbol $\preceq$ is interpreted by the usual order on the set $\{1, \ldots, n\}$, and we speak of first-order formulae over words. For $w \in \Gamma^{*}$ and a first-order sentence over words $\varphi$ we write $w \models \varphi$ if $D_{w}=\varphi$. Throughout this section, let $\Gamma=\{\alpha, \beta\}$ be an alphabet with two elements. The first-order theory of $\Gamma^{*}$ is the set of all first-order sentences over words $\varphi$ such that $w \models \varphi$ for all $w \in \Gamma^{*}$. It is known that the first-order theory of $\Gamma^{*}$ is decidable but not elementary. This lower bound was announced in [47] where it is attributed to Stockmeyer. Stockmeyer's proof can only be found in his thesis and the same applies to the sharpening by Führer while Robertson's independent proof appeared as an extended abstract, only. Complete proofs can be found for instance in $[11,45,58]$.

In a first step we reduce the first-order theory of $\Gamma^{*}$ to the first-order theory of a structure $\left(\mathbb{M}\left(\Sigma_{1}, I_{1}\right), \rightarrow_{\mathcal{R}_{1}},(\langle t\rangle)_{t \in F}\right)$ where $F \subseteq \mathbb{M}\left(\Sigma_{1}, I_{1}\right)$ is finite and $\langle t\rangle=$ $\mathbb{M} t \mathbb{M}$ is the set of all traces that contain the factor $t$. We will achieve this by translating an internal first-order property of a word $w \in \Gamma^{*}$ into an external property of a small sphere around $w$ with respect to a suitable trace rewriting system. This proceeding is in some sense inverse to the internalization technique from the previous section.

Lemma 6.1. There exist an independence alphabet $\left(\Sigma_{1}, I_{1}\right)$ and a trace rewriting system $\mathcal{R}_{1}$ over $\mathbb{M}\left(\Sigma_{1}, I_{1}\right)$ such that the first-order theory of $\Gamma^{*}$ can be reduced in polynomial time to the first-order theory of $\left(\mathbb{M}\left(\Sigma_{1}, I_{1}\right), \rightarrow_{\mathcal{R}_{1}},(\langle t\rangle)_{t \in F}\right)$ for some finite subset $F \subseteq \mathbb{M}\left(\Sigma_{1}, I_{1}\right)$. Moreover the graph $\left(\mathbb{M}\left(\Sigma_{1}, I_{1}\right), \rightarrow_{\mathcal{R}_{1}}\right)$ is acyclic.

Proof. Let $\Sigma_{1}=\Gamma \cup \Gamma^{\prime} \cup\{\$\}$, where $\Gamma^{\prime}=\left\{\alpha^{\prime}, \beta^{\prime}\right\}$ is a disjoint copy of $\Gamma$. On this set, we define an independence relation $I_{1}$ by $I_{1}=(\{\$\} \times \Gamma) \cup(\Gamma \times\{\$\})$. The trace rewriting system $\mathcal{R}_{1}$ is

$$
\mathcal{R}_{1}=\left\{a \rightarrow a^{\prime}, a^{\prime} \rightarrow a^{\prime} \$ \mid a \in \Gamma\right\}
$$

Note that $\left(\mathbb{M}\left(\Sigma_{1}, I_{1}\right), \rightarrow_{\mathcal{R}_{1}}\right)$ is acyclic. Let $F=\Gamma^{\prime} \cup\{\$\} \cup\left\{\$ a^{\prime} \mid a \in \Gamma\right\}$. Finally, let $\varphi$ be a first-order sentence over words. Then it belongs to the theory of $\Gamma^{*}$ if and only if every word $w$ satisfies $\varphi$. Since $\Gamma^{\prime} \cup\{\$\} \subseteq F$, the formula $\bigwedge_{c \in \Gamma^{\prime} \cup\{\$\}} x \notin\langle c\rangle$ is satisfied in $\left(\mathbb{M}\left(\Sigma_{1}, I_{1}\right), \rightarrow_{\mathcal{R}_{1}},(\langle t\rangle)_{t \in F}\right)$ by a word $x$ if and only if $x$ is actually a word over $\Gamma$. For such a trace $x=a_{1} a_{2} \cdots a_{k}$, we will now express that it satisfies $\varphi$. 
First note that the immediate successors of $x$ with respect to the relation $\rightarrow_{\mathcal{R}_{1}}$ are of the form $a_{1} a_{2} \ldots a_{i-1} a_{i}^{\prime} a_{i+1} \cdots a_{k}$. Hence there is a bijection pos from $\left\{y \mid x \rightarrow_{\mathcal{R}_{1}} y\right\}$ to the set of positions $\{1,2, \ldots, k\}$ in $x: \operatorname{pos}\left(a_{1} \cdots a_{i-1} a_{i}^{\prime} a_{i+1} \cdots a_{k}\right)=i$. The position $\operatorname{pos}(y)$ carries the letter $a$ if and only if $y \in\left\langle a^{\prime}\right\rangle$. It remains to express $\operatorname{pos}(y)<$ $\operatorname{pos}(z)$. For this, let us fix $y=a_{1} \cdots a_{i-1} a_{i}^{\prime} a_{i+1} \cdots a_{k}$ and $z=a_{1} \cdots a_{j-1} a_{j}^{\prime} a_{j+1} \cdots a_{k}$. Thus, we have to express $i<j$. Let

$$
\operatorname{less}(y, z) \equiv \exists p \exists q \exists r\left\{\begin{array}{l}
y \rightarrow_{\mathcal{R}_{1}} p \rightarrow_{\mathcal{R}_{1}} r \wedge z \rightarrow_{\mathcal{R}_{1}} q \rightarrow_{\mathcal{R}_{1}} r \wedge \\
p \in\langle \$\rangle \wedge \bigvee_{a \in \Gamma} r \in\left\langle \$ a^{\prime}\right\rangle
\end{array}\right\}
$$

We claim that $i<j$ if and only if $\operatorname{less}(y, z)$. If $i<j$ then we can take

$$
\begin{aligned}
& p=a_{1} \cdots a_{i-1} a_{i}^{\prime} \$ a_{i+1} \cdots a_{k} \\
& r=a_{1} \cdots a_{i-1} a_{i}^{\prime} a_{i+1} \cdots a_{j-1} \$ a_{j}^{\prime} a_{j+1} \cdots a_{k} \\
& q=a_{1} \cdots a_{i-1} a_{i}^{\prime} a_{i+1} \cdots a_{j-1} a_{j}^{\prime} a_{j+1} \cdots a_{k}
\end{aligned}
$$

On the other hand, assume that $\operatorname{less}(y, z)$ holds. Since $y \rightarrow_{\mathcal{R}_{1}} p$ and $p \in\langle \$\rangle$ we must have $p=a_{1} \cdots a_{i-1} a_{i}^{\prime} \$ a_{i+1} \cdots a_{k}$. Since $p \rightarrow_{\mathcal{R}_{1}} r$ and $\bigvee_{a \in \Gamma} r \in\left\langle \$ a^{\prime}\right\rangle$ there exists $\ell>i$ with $r=a_{1} \cdots a_{i-1} a_{i}^{\prime} a_{i+1} \cdots a_{\ell-1} \$ a_{\ell}^{\prime} a_{\ell+1} \cdots a_{k}$ Finally, $z \rightarrow_{\mathcal{R}_{1}} q \rightarrow_{\mathcal{R}_{1}} r$ implies that $\ell=j$, thus $i<j$.

Recall that $\varphi$ is a first-order formula over words. Let $\psi(x)$, where $x$ is a variable which does not occur in $\varphi$, be derived from $\varphi$ by inductively

- replacing any subformula of the form $\exists y \gamma$ by $\exists y\left(x \rightarrow_{\mathcal{R}_{1}} y \wedge \gamma\right)$,

- replacing any subformula of the form $y<z$ by $\operatorname{less}(y, z)$, and

- replacing any subformula of the form $Q_{a}(y)$ by $y \in\left\langle a^{\prime}\right\rangle$.

By our considerations above, for a word $w \in \Gamma^{*}$ we have

$$
\left(\mathbb{M}\left(\Sigma_{1}, I_{1}\right), \rightarrow_{\mathcal{R}_{1}},(\langle t\rangle)_{t \in F}\right) \models \psi(w) \quad \text { if and only if } \quad w \models \varphi .
$$

Hence $\varphi$ belongs to the first-order theory of $\Gamma^{*}$ if and only if

$$
\left(\mathbb{M}\left(\Sigma_{1}, I_{1}\right), \rightarrow_{\mathcal{R}_{1}},(\langle t\rangle)_{t \in F}\right) \models \forall x\left\{\bigwedge_{c \in \Gamma^{\prime} \cup\{\$\}} x \notin\langle c\rangle \Rightarrow \psi(x)\right\} .
$$

Lemma 6.2. Let $\mathcal{R}_{1}$ be a trace rewriting system over $\mathbb{M}\left(\Sigma_{1}, I_{1}\right)$ such that the graph $\left(\mathbb{M}\left(\Sigma_{1}, I_{1}\right), \rightarrow_{\mathcal{R}_{1}}\right)$ is acyclic. Let $F \subseteq \mathbb{M}\left(\Sigma_{1}, I_{1}\right)$ be finite. Then there is an independence alphabet $(\Sigma, I)$ and a trace rewriting system $\mathcal{R}$ such that the first-order theory of $\left(\mathbb{M}\left(\Sigma_{1}, I_{1}\right), \rightarrow_{\mathcal{R}_{1}},(\langle t\rangle)_{t \in F}\right)$ can be reduced in polynomial time to the first-order theory of $\left(\mathbb{M}(\Sigma, I), \rightarrow_{\mathcal{R}}\right)$. 
Proof. Let $F=\left\{t_{1}, \ldots, t_{n}\right\}$. Then define

$$
\Sigma=\Sigma_{1} \dot{\cup}\left\{(k, i) \in \mathbb{N}^{2} \mid 1 \leq i \leq n, 0 \leq k<i\right\}
$$

where $\dot{\cup}$ denotes disjoint union. Consider $I_{1} \subseteq \Sigma_{1} \times \Sigma_{1}$ as an independence relation $I \subseteq \Sigma \times \Sigma$. We consider the following trace rewriting system $\mathcal{R}$ over $\mathbb{M}(\Sigma, I)$ :

$$
\begin{aligned}
\mathcal{R}_{1} & \cup\left\{t_{i} \rightarrow(0, i) \mid 1 \leq i \leq n\right\} \\
& \cup\{(k, i) \rightarrow((k+1) \bmod i, i) \mid 1 \leq i \leq n, 0 \leq k<i\} .
\end{aligned}
$$

Intuitively, the graph $\left(\mathbb{M}(\Sigma, I), \rightarrow_{\mathcal{R}}\right)$ is obtained from the graph $\left(\mathbb{M}\left(\Sigma_{1}, I_{1}\right), \rightarrow_{\mathcal{R}_{1}}\right)$ by the addition of cycles of length $i$ to any element of $\left\langle t_{i}\right\rangle$. More precisely, we observe the following:

- A trace $t \in \mathbb{M}(\Sigma, I)$ contains a letter from $\Sigma \backslash \Sigma_{1}$ if and only if it lies on some cycle of length at most $n$. Hence the set of traces $\mathbb{M}\left(\Sigma_{1}, I_{1}\right) \subseteq \mathbb{M}(\Sigma, I)$ can be defined in the graph $\left(\mathbb{M}(\Sigma, I), \rightarrow_{\mathcal{R}}\right)$.

- A trace $t \in \mathbb{M}\left(\Sigma_{1}, I_{1}\right)$ belongs to $\left\langle t_{i}\right\rangle$ if and only if, in the graph $\left(\mathbb{M}(\Sigma, I), \rightarrow_{\mathcal{R}}\right)$, it has an immediate successor that lies on a cycle of length $i$. Hence, also the set $\left\langle t_{i}\right\rangle \subseteq \mathbb{M}\left(\Sigma_{1}, I_{1}\right)$ can be defined in the graph $\left(\mathbb{M}(\Sigma, I), \rightarrow_{\mathcal{R}}\right)$.

Theorem 6.3. There exist an independence alphabet $(\Sigma, I)$ and a trace rewriting system $\mathcal{R}$ over $\mathbb{M}(\Sigma, I)$ such that the first-order theory of $\left(\mathbb{M}(\Sigma, I), \rightarrow_{\mathcal{R}}\right)$ is not elementarily decidable.

Proof. The result follows from the two preceding lemmas and the nonelementary complexity of the first-order theory of $\Gamma^{*}$.

Note that the proof of Lemma 6.1 required a nontrivial independence alphabet. This allowed to relate positions $i$ and $j$ by shifting $\$$ from position $i$ to $j$. Next we want to prove a lower bound for the first-order theory of semi-Thue systems where this trick is not available. Note that the procedure from $[14,29]$ is not elementary since it relies on the complementation of finite automata for any quantifier alternation. Nevertheless, we are only able to show an elementary lower bound, namely doubly exponential nondeterministic time. This is achieved by reducing the successor theory of $\Gamma^{*}$. This theory is defined in the same way as the first-order theory of $\Gamma^{*}$, but the order $<$ on $\{1, \ldots, n\}$ is replaced by the successor-relation suc $=\{(i, i+1) \mid 0 \leq i<n\}$. By [11, Example 8.6], its complexity is at least doubly exponential nondeterministic time.

Lemma 6.4. There exist an alphabet $\Sigma_{1}$ and a semi-Thue system $\mathcal{R}_{1}$ over $\Sigma_{1}^{*}$ such that the successor theory of $\Gamma^{*}$ can be reduced in polynomial time to the first-order theory of $\left(\Sigma_{1}^{*}, \rightarrow_{\mathcal{R}_{1}},(\langle t\rangle)_{t \in F}\right)$ for some finite set $F \subseteq \Sigma_{1}^{*}$. Moreover the graph $\left(\Sigma_{1}^{*}, \rightarrow_{\mathcal{R}_{1}}\right)$ is acyclic.

Proof. We can reuse the construction from the proof of Lemma 6.1, except that we have to set $I_{1}=\emptyset$. Thus, $\mathcal{R}_{1}$ becomes a semi-Thue system over $\mathbb{M}\left(\Sigma_{1}, I_{1}\right)=\Sigma_{1}^{*}$. Then we have less $(y, z)$ if and only if $\operatorname{pos}(z)=\operatorname{pos}(y)+1$. 
Theorem 6.5. There exist an alphabet $\Sigma$ and a semi-Thue system $\mathcal{R}$ over $\Sigma^{*}$ such that any decision procedure for the first-order theory of $\left(\Sigma^{*}, \rightarrow_{\mathcal{R}}\right)$ requires at least doubly exponential nondeterministic time.

Proof. Note that in the proof of Lemma 6.2 we only added letters that are dependent from any other letter. Hence the result follows from Lemma 6.4, Lemma 6.2 and [11, Example 8.6].

\section{Applications to the confluence problem}

In this section we will present some applications of Corollary 3.9 to the confluence problem for trace rewriting systems. Let us fix a trace rewriting system $\mathcal{R}$ over a trace monoid $\mathbb{M}=\mathbb{M}(\Sigma, I)$. We say that $\mathcal{R}$ is confluent if for all $u, v_{1}, v_{2} \in \mathbb{M}$ with

$u \stackrel{*}{\rightarrow} \mathcal{R} v_{1}$ and $u \stackrel{*_{\rightarrow}}{\mathcal{R}} v_{2}$ there exists $w \in \mathbb{M}$ with $v_{1} \stackrel{*}{\rightarrow}_{\mathcal{R}} w$ and $v_{2} \stackrel{*}{\rightarrow}_{\mathcal{R}} w$. If for $u \in \mathbb{M}$ there does not exist an infinite derivation $u=u_{1} \rightarrow_{\mathcal{R}} u_{2} \rightarrow_{\mathcal{R}} u_{3} \rightarrow_{\mathcal{R}} \cdots$ then $\mathcal{R}$ is called terminating on $u$. If $\mathcal{R}$ is terminating on all $u \in \mathbb{M}$ then $\mathcal{R}$ is terminating. If $\mathcal{R}$ is terminating then, by Newman's Lemma [52], $\mathcal{R}$ is confluent if and only if $\mathcal{R}$ is locally confluent, see the definition before Lemma 3.10. We say that $\mathcal{R}$ is lengthreducing if $|\ell|>|r|$ for every $(\ell, r) \in \mathcal{R}$. Obviously, if $\mathcal{R}$ is length reducing then $\mathcal{R}$ is also terminating. As already noted in the proof of Lemma 3.10 every semi-Thue system has only finitely many so called critical pairs. In order to check local confluence for semi-Thue systems, only these critical pairs have to be considered. Thus, Newman's Lemma implies that confluence can be decided for terminating semi-Thue systems, see e.g. [4]. Below we will generalize the notion of a critical pair to trace rewriting systems. But in contrast to semi-Thue systems, a trace rewriting system has in general infinitely many critical pairs. This is an unavoidable phenomenon, as the next theorem from [40] shows. It generalizes a previous result of [51]:

Theorem 7.1. Let $(\Sigma, I)$ be an independence alphabet. The following problem is decidable if and only if $I=\emptyset$ or $I=(\Sigma \times \Sigma) \backslash I d_{\Sigma}$ :

INPUT: A length-reducing trace rewriting system $\mathcal{R}$ over $\mathbb{M}(\Sigma, I)$

QUESTION: Is $\mathcal{R}$ confluent?

Hence in almost all cases confluence is undecidable for length-reducing trace rewriting systems. On the other hand, in $[16,40]$ several subclasses of trace rewriting systems were defined for which confluence becomes decidable. In this section we define a further class of trace rewriting systems with a decidable confluence problem. We remark that this class is not contained in the classes defined in $[16,40]$. The main idea is to give sufficient conditions which imply the equivalence of confluence and $\alpha$-confluence (see Section 3.3) for some $\alpha>0$ that can be calculated effectively. This makes it possible to apply the following immediate corollary of Corollary 3.9.

Corollary 7.2. The following problem is decidable:

INPUT: A trace rewriting system $\mathcal{R}$ and $\alpha \in \mathbb{N}$.

QUESTION: Is $\mathcal{R} \alpha$-confluent? 
Before we present our announced decidability result, we first have to introduce some notation. For a trace $u \in \mathbb{M}$ we define $\mathrm{D}(u)=\{a \in \Sigma \mid(a, u) \notin I\}$. In [40] the second author has introduced the following condition for trace rewriting systems: $\mathcal{R}$ satisfies condition (A) if the following holds:

(A1) For all $(\ell, r) \in \mathcal{R}$ and all $a \in \Sigma$, if $(a, \ell) \in I$ then $a r=r a$.

(A2) For all $p_{0}, p_{1}, q_{0}, q_{1} \in \mathbb{M} \backslash\{1\}, r_{0}, r_{1} \in \mathbb{M}$ with $\left(p_{0} q_{0}, r_{0}\right),\left(p_{1} q_{1}, r_{1}\right) \in \mathcal{R}$ and $\left(p_{0}, p_{1}\right),\left(q_{0}, q_{1}\right) \in I$ there exist $s_{0}, s_{1}, t_{0}, t_{1} \in \mathbb{M}$ such that $r_{i}=s_{i} t_{i}, \mathrm{D}\left(s_{i}\right) \subseteq$ $\mathrm{D}\left(p_{i}\right)$, and $\mathrm{D}\left(t_{i}\right) \subseteq \mathrm{D}\left(q_{i}\right)$ for $i=0,1$.

The set $\operatorname{CS}(\mathcal{R})$ of all critical situations of $\mathcal{R}$ is the set of all triples $\left(t_{0}, t, t_{1}\right)$ such that there exist traces $p_{0}, p_{1}, q_{0}, q_{1}, w_{0}, w_{1}, r_{0}, r_{1} \in \mathbb{M}, s \in \mathbb{M} \backslash\{1\}$ with

(CS1) $\left(p_{0} s q_{0}, r_{0}\right),\left(p_{1} s q_{1}, r_{1}\right) \in \mathcal{R}$,

(CS2) $\left(p_{0}, p_{1}\right),\left(q_{0}, q_{1}\right),\left(w_{0}, w_{1}\right),\left(s, w_{0} w_{1}\right),\left(w_{0}, q_{0} p_{1}\right),\left(w_{1}, p_{0} q_{1}\right) \in I$, and

(CS3) $t=p_{1} w_{1} p_{0} s q_{0} w_{0} q_{1}=p_{0} w_{0} p_{1} s q_{1} w_{1} q_{0},{ }^{10} t_{0}=p_{1} w_{1} r_{0} w_{0} q_{1}, t_{1}=p_{0} w_{0} r_{1} w_{1} q_{0}$.

Note that for all $\left(t_{0}, t, t_{1}\right) \in \operatorname{CS}(\mathcal{R}), t \rightarrow_{\mathcal{R}} t_{0}$ and $t \rightarrow_{\mathcal{R}} t_{1}$. Furthermore note that a trace rewriting system has in general infinitely many critical situations.

Lemma 7.3. Let $\mathcal{R}$ satisfy condition (A). Then $\mathcal{R}$ is $\alpha$-confluent if and only if for every $\left(u_{0}, u, u_{1}\right) \in C S(\mathcal{R})$ there exists $v \in \mathbb{M}$ with $u_{0} \rightarrow \underset{\mathcal{R}}{\leq \alpha} v$ and $u_{1} \rightarrow \underset{\mathcal{R}}{\leq \alpha} v$.

Proof. This can be shown precisely as in the proof of [40, Lemma 2.3]. There, an additional condition is present in the definition of critical situations. This condition is not needed for our purpose, and it does not influence the proof.

For a subalphabet $\Gamma \subseteq \Sigma$ we define the rewriting system $\pi_{\Gamma}(\mathcal{R})$ by

$$
\pi_{\Gamma}(\mathcal{R})=\left\{\left(\pi_{\Gamma}(\ell), \pi_{\Gamma}(r)\right) \mid(\ell, r) \in \mathcal{R}\right\}
$$

Theorem 7.4. The following problem is decidable.

INPUT: A trace rewriting system $\mathcal{R}$ over the trace monoid $\mathbb{M}(\Sigma, I)$ with the following properties:

- $\mathcal{R}$ satisfies condition $(A)$ and is terminating.

- For all $p_{0}, p_{1}, q_{0}, q_{1}, r_{0}, r_{1} \in \mathbb{M}, s \in \mathbb{M} \backslash\{1\}$ with $\left(p_{0} s q_{0}, r_{0}\right),\left(p_{1} s q_{1}, r_{1}\right) \in \mathcal{R}$ and $\left(p_{0}, p_{1}\right),\left(q_{0}, q_{1}\right) \in I$, the rewriting system $\pi_{\Gamma}(\mathcal{R})$ is terminating on the traces $\pi_{\Gamma}\left(p_{1} r_{0} q_{1}\right)$ and $\pi_{\Gamma}\left(p_{0} r_{1} q_{0}\right)$ where $\Gamma=\mathrm{D}\left(p_{0} s q_{1}\right) \cap \mathrm{D}\left(p_{1} s q_{0}\right) \subseteq \Sigma$.

QUESTION: Is $\mathcal{R}$ confluent?

Proof. Let $\mathcal{R}$ be a trace rewriting system which satisfies the conditions from the theorem. We prove the theorem by effectively calculating an $\alpha>0$ such that $\mathcal{R}$ is confluent if and only if $\mathcal{R}$ is $\alpha$-confluent. This allows us to apply Corollary 7.2.

\footnotetext{
${ }^{10}$ Note that the equality of these two factorizations of $t$ follows from the independencies listed in (CS2).
} 
For all $p_{0}, p_{1}, q_{0}, q_{1}, r_{0}, r_{1} \in \mathbb{M}, s \in \mathbb{M} \backslash\{1\}$ with $\left(p_{0} s q_{0}, r_{0}\right),\left(p_{1} s q_{1}, r_{1}\right) \in \mathcal{R}$ and $\left(p_{0}, p_{1}\right),\left(q_{0}, q_{1}\right) \in I$ we define a natural number $\alpha\left(p_{0}, p_{1}, q_{0}, q_{1}, r_{0}, r_{1}, s\right)$ by

$$
\alpha\left(p_{0}, p_{1}, q_{0}, q_{1}, r_{0}, r_{1}, s\right)=\max \left\{k \mid \exists u\left(\begin{array}{ll}
\pi_{\Gamma}\left(p_{1} r_{0} q_{1}\right) \rightarrow_{\pi_{\Gamma}(\mathcal{R})}^{k} \pi_{\Gamma}(u) & \text { or } \\
\pi_{\Gamma}\left(p_{0} r_{1} q_{0}\right) \rightarrow_{\pi_{\Gamma}(\mathcal{R})}^{k} \pi_{\Gamma}(u)
\end{array}\right)\right\}
$$

where $\Gamma=\mathrm{D}\left(p_{0} s q_{1}\right) \cap \mathrm{D}\left(p_{1} s q_{0}\right) \subseteq \Sigma$. The assumptions on $\mathcal{R}$ imply that the number $\alpha\left(p_{0}, p_{1}, q_{0}, q_{1}, r_{0}, r_{1}, s\right)$ can be calculated effectively. Now let

$$
\alpha=\max \left\{\begin{array}{l|l}
\alpha\left(p_{0}, p_{1}, q_{0}, q_{1}, r_{0}, r_{1}, s\right) & \begin{array}{l}
\left(p_{0} s q_{0}, r_{0}\right),\left(p_{1} s q_{1}, r_{1}\right) \in \mathcal{R}, \\
\left(p_{0}, p_{1}\right),\left(q_{0}, q_{1}\right) \in I, s \neq 1
\end{array}
\end{array} .\right.
$$

Also $\alpha$ can be calculated effectively. We claim that $\mathcal{R}$ is confluent if and only if $\mathcal{R}$ is $\alpha$-confluent. If $\mathcal{R}$ is $\alpha$-confluent then $\mathcal{R}$ is also locally confluent. Since $\mathcal{R}$ is terminating, $\mathcal{R}$ must be confluent. Now assume that $\mathcal{R}$ is confluent. We claim that $\mathcal{R}$ is $\alpha$-confluent. By Lemma 7.3 it suffices to consider all critical situations of $\mathcal{R}$. Let $\left(t_{0}, t, t_{1}\right) \in \mathrm{CS}(\mathcal{R})$. By the definition of critical situations there exist traces $p_{0}, p_{1}, q_{0}$, $q_{1}, w_{0}, w_{1}, r_{0}, r_{1} \in \mathbb{M}$, and $s \in \mathbb{M} \backslash\{1\}$ such that (CS1), (CS2), and (CS3) hold. Since $t \rightarrow_{\mathcal{R}} t_{0}, t \rightarrow_{\mathcal{R}} t_{1}$, and $\mathcal{R}$ is confluent, there exist $\alpha_{0}, \alpha_{1} \geq 0$ and a trace $u \in \mathbb{M}(\Sigma, I)$ such that

$$
t_{0}=p_{1} w_{1} r_{0} w_{0} q_{1} \rightarrow_{\mathcal{R}}^{\alpha_{0}} u \quad \text { and } \quad t_{1}=p_{0} w_{0} r_{1} w_{1} q_{0} \rightarrow_{\mathcal{R}}^{\alpha_{1}} u
$$

Let $\Gamma=\mathrm{D}\left(p_{0} s q_{1}\right) \cap \mathrm{D}\left(p_{1} s q_{0}\right)$. From (CS2) we obtain $\left(w_{0}, p_{1} s q_{0}\right),\left(w_{1}, p_{0} s q_{1}\right) \in I$. Thus $\pi_{\Gamma}\left(w_{0}\right)=1=\pi_{\Gamma}\left(w_{1}\right)$. Hence (13) implies

$$
\pi_{\Gamma}\left(p_{1} r_{0} q_{1}\right) \rightarrow_{\pi_{\Gamma}(\mathcal{R})}^{\alpha_{0}} \pi_{\Gamma}(u) \quad \text { and } \quad \pi_{\Gamma}\left(p_{0} r_{1} q_{0}\right) \rightarrow_{\pi_{\Gamma}(\mathcal{R})}^{\alpha_{1}} \pi_{\Gamma}(u) .
$$

The definition of $\alpha$ and (CS1) and (CS2) implies that $\alpha_{0}, \alpha_{1} \leq \alpha$. Finally Lemma 7.3 implies that $\mathcal{R}$ is $\alpha$-confluent.

The decidability criterion from Theorem 7.4 is quite technical. In the following we will present two less technical decidability criteria which can be deduced from Theorem 7.4. The following theorem can also be found in [38].

Theorem 7.5. The following problem is decidable:

INPUT: A trace rewriting system $\mathcal{R}$ over $\mathbb{M}(\Sigma, I)$ such that there exists a clique covering $\left(\Gamma_{1}, \ldots, \Gamma_{n}\right)$ of the dependence alphabet $(\Sigma, D)$ (where $D=(\Sigma \times \Sigma) \backslash I$ ) with the following properties:

(1) For all rules $(\ell, r) \in \mathcal{R}$ and all $i, j \in\{1, \ldots, n\}$ with $i \neq j$ and $\pi_{i}\left(\pi_{j}(\ell)\right)=\epsilon$, we have $\pi_{i}\left(\pi_{j}(r)\right)=\epsilon$.

(2) For all $i \in\{1, \ldots, n\}$, the semi-Thue system $\pi_{i}(\mathcal{R})=\pi_{\Gamma_{i}}(\mathcal{R})$ is terminating.

QUESTION: Is $\mathcal{R}$ confluent? 
Proof. Let $\mathcal{R}$ be a trace rewriting system over $\mathbb{M}(\Sigma, I)$, and let $\left(\Gamma_{1}, \ldots, \Gamma_{n}\right)$ be a clique covering of the dependence alphabet $(\Sigma, D)$ which satisfies (1) and (2) from the theorem. We will show that $\mathcal{R}$ satisfies the conditions on the input in Theorem 7.4.

Condition (2) obviously implies that $\mathcal{R}$ is terminating. Next we show that $\mathcal{R}$ satisfies condition (A). Let $\ell$ be a left-hand side of a rule in $\mathcal{R}$. Condition (2) implies $\pi_{i}(\ell) \neq \epsilon$ for all $i \in\{1, \ldots, n\}$, because otherwise $\pi_{i}(\mathcal{R})$ would contain a rule of the form $\epsilon \rightarrow \pi_{i}(r)$ and would therefore not be terminating. Thus there cannot exist $a \in \Sigma$ with $(a, \ell) \in I$, i.e., $\mathcal{R}$ satisfies condition (A1). Condition (A2) can be verified as follows. Let $\left(p_{0} q_{0}, r_{0}\right),\left(p_{1} q_{1}, r_{1}\right) \in \mathcal{R}$ such that $1 \notin\left\{p_{0}, p_{1}, q_{0}, q_{1}\right\}$ and $\left(p_{0}, p_{1}\right),\left(q_{0}, q_{1}\right) \in I$. We have to find factorizations $r_{0}=s_{0} t_{0}$ and $r_{1}=s_{1} t_{1}$ such that $\mathrm{D}\left(s_{j}\right) \subseteq \mathrm{D}\left(p_{j}\right)$ and $\mathrm{D}\left(t_{j}\right) \subseteq \mathrm{D}\left(q_{j}\right)$ for $j=0,1$. First note that for arbitrary traces $u, v$, if $(u, v) \in I$ then $\pi_{i}(u)=\epsilon$ or $\pi_{i}(v)=\epsilon$ for every clique $\Gamma_{i}$. Since furthermore $\pi_{i}\left(p_{0} q_{0}\right) \neq \epsilon \neq \pi_{i}\left(p_{1} q_{1}\right)$, for all $i \in\{1, \ldots, n\}$ exactly one of the following two cases holds:

- case $1 . \pi_{i}\left(p_{0}\right) \neq \epsilon \neq \pi_{i}\left(q_{1}\right)$ and $\pi_{i}\left(p_{1}\right)=\epsilon=\pi_{i}\left(q_{0}\right)$

- case 2. $\pi_{i}\left(p_{1}\right) \neq \epsilon \neq \pi_{i}\left(q_{0}\right)$ and $\pi_{i}\left(p_{0}\right)=\epsilon=\pi_{i}\left(q_{1}\right)$

Now we define for every $i \in\{1, \ldots, n\}$ a factorization $\pi_{i}\left(r_{0}\right)=u_{i} v_{i}$ of the word $\pi_{i}\left(r_{0}\right)$ as follows:

- $u_{i}=\pi_{i}\left(r_{0}\right)$ and $v_{i}=\epsilon$ if case 1 holds for $i$.

- $u_{i}=\epsilon$ and $v_{i}=\pi_{i}\left(r_{0}\right)$ if case 2 holds for $i$.

We claim that the tuples $\left(u_{1}, \ldots, u_{n}\right)$ and $\left(v_{1}, \ldots, v_{n}\right)$ are reconstructible (see the definition before Lemma 3.4). It suffices, by Lemma 3.4, to show that $\pi_{i}\left(u_{j}\right)=\pi_{j}\left(u_{i}\right)$ for all $i, j \in\{1, \ldots, n\}$ with $i \neq j$. So let $i, j \in\{1, \ldots, n\}$. If $i$ and $j$ both satisfy case 1 or $i$ and $j$ both satisfy case 2 , then this holds obviously. Thus w.l.o.g. let us assume that $i$ satisfies case 1 and $j$ satisfies case 2. This implies $u_{i}=\pi_{i}\left(r_{0}\right), u_{j}=\epsilon$ and

$$
\begin{array}{ll}
\pi_{i}\left(p_{0}\right) \neq \epsilon \neq \pi_{i}\left(q_{1}\right), & \pi_{i}\left(p_{1}\right)=\epsilon=\pi_{i}\left(q_{0}\right), \\
\pi_{j}\left(p_{1}\right) \neq \epsilon \neq \pi_{j}\left(q_{0}\right), & \pi_{j}\left(p_{0}\right)=\epsilon=\pi_{j}\left(q_{1}\right) .
\end{array}
$$

Therefore $\pi_{i}\left(\pi_{j}\left(p_{0} q_{0}\right)\right)=\epsilon=\pi_{i}\left(\pi_{j}\left(p_{1} q_{1}\right)\right)$. Condition (1) implies that $\pi_{i}\left(\pi_{j}\left(r_{0}\right)\right)=$ $\epsilon=\pi_{i}\left(\pi_{j}\left(r_{1}\right)\right)$. Thus $\pi_{i}\left(u_{j}\right)=\pi_{i}(\epsilon)=\epsilon=\pi_{j}\left(\pi_{i}\left(r_{0}\right)\right)=\pi_{j}\left(u_{i}\right)$. We have shown that the tuples $\left(u_{1}, \ldots, u_{n}\right)$ and $\left(v_{1}, \ldots, v_{n}\right)$ are reconstructible. Thus there exist unique traces $s_{0}$ and $t_{0}$ with $\pi_{i}\left(s_{0}\right)=u_{i}$ and $\pi_{i}\left(t_{0}\right)=v_{i}$ for all $i \in\{1, \ldots, n\}$. Lemma 3.3 implies $r_{0}=s_{0} t_{0}$.

Now assume that $a \in \mathrm{D}\left(s_{0}\right)$. Thus there exists $j \in\{1, \ldots, n\}$ such that $a \in \Gamma_{j}$ and $u_{j}=\pi_{j}\left(s_{0}\right) \neq \epsilon$. Thus case 1 from the construction of $\left(u_{1}, \ldots, u_{n}\right)$ holds for $j$ which implies that $\pi_{j}\left(p_{0}\right) \neq \epsilon$, i.e., $a \in D\left(p_{0}\right)$. Analogously it can be shown that $\mathrm{D}\left(t_{0}\right) \subseteq \mathrm{D}\left(q_{0}\right)$. Thus we have constructed the desired factorization of $r_{0}$. The desired factorization $r_{1}=s_{1} t_{1}$ of $r_{1}$ can be constructed analogously. Thus $\mathcal{R}$ satisfies condition (A2) and hence condition (A).

In order to show the second condition from Theorem 7.4, let us consider rules $\left(p_{0} s q_{0}, r_{0}\right),\left(p_{1} s q_{1}, r_{1}\right) \in \mathcal{R}$ with $\left(p_{0}, p_{1}\right),\left(q_{0}, q_{1}\right) \in I$ and $s \neq 1$. Let $\Gamma=\mathrm{D}\left(p_{0} s q_{1}\right) \cap$ 
$\mathrm{D}\left(p_{1} s q_{0}\right)$. Because of $s \neq 1$ there exists a clique $\Gamma_{i}$ with $\Gamma_{i} \subseteq \mathrm{D}(s) \subseteq \Gamma$. Since $\pi_{i}(\mathcal{R})$ is terminating, also $\pi_{\Gamma}(\mathcal{R})$ is terminating which concludes the proof.

Example 7.6. Let $\mathcal{R}$ be a trace rewriting system over a direct product $\prod_{i=1}^{n} \Gamma_{i}^{*}$ of free monoids. If each of the semi-Thue systems $\pi_{\Gamma_{i}}(\mathcal{R})$ is terminating, $i \in\{1, \ldots, n\}$, then we can apply Theorem 7.5 in order to check whether $\mathcal{R}$ is confluent. In particular this is true if $\mathcal{R}$ is length-reducing in each of its $n$ components. On the other hand, confluence is undecidable for the class of all rewriting systems over $\{a, b\}^{*} \times\{c\}^{*}$ for which all rules are length-preserving in one component but length-reducing in the other component (this follows immediately from the construction given in the proof of [40, Lemma 3.4]). This gives a very sharp borderline between decidability and undecidability for the case of direct products of free monoids.

The statement of the following theorem is similar to that of Theorem 7.5. A trace $t \in \mathbb{M}$ is connected if there does not exist a factorization $t=t_{1} t_{2}$ such that $t_{1} \neq 1 \neq t_{2}$ and $\left(t_{1}, t_{2}\right) \in I$.

Theorem 7.7. The following problem is decidable:

INPUT: A trace rewriting system $\mathcal{R}$ over $\mathbb{M}(\Sigma, I)$ such that

(1) for all $(\ell, r) \in \mathcal{R}$ the trace $\ell$ is connected, and

(2) there exists a clique covering $\left(\Gamma_{1}, \ldots, \Gamma_{n}\right)$ of $(\Sigma, D)$ (where $D=(\Sigma \times \Sigma) \backslash I$ ) such that for all $i \in\{1, \ldots, n\}$ the semi-Thue system $\pi_{i}(\mathcal{R})=\pi_{\Gamma_{i}}(\mathcal{R})$ is terminating.

QUESTION: Is $\mathcal{R}$ confluent?

Proof. The proof is quite similar to the proof of Theorem 7.5. Let $\mathcal{R}$ be a trace rewriting system which satisfies condition (1) and (2). Let $\left(\Gamma_{1}, \ldots, \Gamma_{n}\right)$ be a clique covering of the dependence alphabet $(\Sigma, D)$ such that for all $i \in\{1, \ldots, n\}$ the semi-Thue system $\pi_{i}(\mathcal{R})$ is terminating. Thus $\mathcal{R}$ must be terminating. Analogously to the proof of Theorem 7.5 it can be shown that $\mathcal{R}$ satisfies the second condition from Theorem 7.4. Furthermore $\pi_{i}(\ell) \neq \epsilon$ holds for all left-hand sides $\ell$ of $\mathcal{R}$ and all $i \in\{1, \ldots, n\}$. Hence $\mathcal{R}$ also satisfies condition (A1). It remains to show that $\mathcal{R}$ satisfies condition (A2). Assume that there exist rules $\left(p_{0} q_{0}, r_{0}\right),\left(p_{1} q_{1}, r_{1}\right) \in \mathcal{R}$ such that $1 \notin\left\{p_{0}, p_{1}, q_{0}, q_{1}\right\}$ and $\left(p_{0}, p_{1}\right),\left(q_{0}, q_{1}\right) \in I$. We will deduce a contradiction. Assume that $\pi_{i}\left(p_{0}\right) \neq \epsilon$ for some $i \in\{1, \ldots, n\}$. Then $\left(p_{0}, p_{1}\right) \in I$ implies $\pi_{i}\left(p_{1}\right)=\epsilon$. Thus $\pi_{i}\left(q_{1}\right)=\pi_{i}\left(p_{1} q_{1}\right) \neq \epsilon$. Finally $\left(q_{0}, q_{1}\right) \in I$ implies $\pi_{i}\left(q_{0}\right)=\epsilon$. We have shown that $\pi_{i}\left(p_{0}\right) \neq \epsilon$ implies $\pi_{i}\left(q_{0}\right)=\epsilon$ for all $i \in\{1, \ldots, n\}$. It follows that $\left(p_{0}, q_{0}\right) \in I$, because otherwise there would exist a clique $\Gamma_{j}$ with $\pi_{j}\left(p_{0}\right) \neq \epsilon \neq \pi_{j}\left(q_{0}\right)$. But because of $p_{0} \neq 1 \neq q_{0}$ this contradicts the assumption that $p_{0} q_{0}$ is connected.

Example 7.8. Let $\Sigma=\{a, b, c, d\}$ and $I=\{(a, d),(d, a),(b, c),(c, b)\}$. Let $\mathcal{R}$ be the trace rewriting system $\{b d c \rightarrow a\}$ over the trace monoid $\mathbb{M}(\Sigma, I)$. A clique covering of the corresponding dependence alphabet is $(\{a, b\},\{b, d\},\{c, d\},\{a, c\})$. The trace $b d c$ is connected and the projection of the rule $b d c \rightarrow a$ onto any of the four cliques is terminating. Therefore we can apply Theorem 7.7 in order to check whether $\mathcal{R}$ 
is confluent (it is easy to see that $\mathcal{R}$ is confluent). Note that here we cannot apply Theorem 7.5 because $\pi_{\{a, b\}}\left(\pi_{\{a, c\}}(b d c)\right)=\epsilon$ and $\pi_{\{a, b\}}\left(\pi_{\{a, c\}}(a)\right) \neq \epsilon$. On the other hand, in general Theorem 7.7 cannot be applied to trace rewriting systems over direct products of free monoids which we have considered in Example 7.6.

\section{Open questions}

In Section 6 we proved a lower bound for the complexity of the first-order theory of the one-step rewrite graph of a semi-Thue system. There is a huge gap between this doubly exponential lower and the nonelementary upper bound that follows immediately from the proofs in $[14,29]$.

Although our main decidability result is very similar to corresponding results in $[14,29]$, our technique is new. It could provide a means to identify term rewriting systems whose rewrite graph has a decidable first-order theory. So far, this property has been shown for quite restricted term rewriting systems only, like ground term rewriting systems [14] and left-linear right-ground systems [64]. On the other hand, Treinen's result [65] shows that not much more can be expected.

Semi-Thue systems can be seen as term rewriting systems modulo associativity (it is a very simple case since there are no further symbols). Similarly, trace rewriting is term rewriting modulo associativity and partial commutativity. Is it possible to use the technique developed in this paper to handle other "term rewriting modulo ..." theories?

In Section 5.5, we considered a counting extension of first-order logic. So far, our technique can deal with local properties of this logic, only. Is the FO+MOD theory of one-step rewriting in general decidable? In order to answer this question positively, one would have to develop another Gaifman Theorem for this counting logic.

As already mentioned in the introduction, the decidability of the first-order theory of the one-step rewrite graph of a semi-Thue system follows from the fact that, in this case, the one-step rewrite relation can be recognized by a two-tape automaton where both heads move synchronously. In other words, the one-step rewrite graph of a semiThue system is an automatic graph [31]. It is known that every automatic graph has a decidable first-order theory [31]. We conjecture that the one-step rewrite graphs of trace rewriting systems are in general not automatic. This might be a difficult problem, since there are only few tools for proving that a given graph is not automatic, see [3], ${ }^{11}$ which seem not to apply in our case.

\section{References}

1. G. Bauer and F. Otto. Finite complete rewriting systems and the complexity of the word problem. Acta Informatica, 21:521-540, 1984.

2. A. Blumensath. Prefix-recognizable graphs and monadic second-order logic. Technical Report 2001-06, RWTH Aachen, Department of Computer Science, 2001.

\footnotetext{
${ }^{11}$ The main problem is that the underlying alphabet can be chosen arbitrary for an automatic graph.
} 
3. A. Blumensath and E. Grädel. Automatic structures. In Proceedings of the 15th Annual IEEE Symposium on Logic in Computer Science (LICS'2000), pages 51-62. IEEE Computer Society Press, 2000.

4. R. V. Book and F. Otto. String-Rewriting Systems. Springer, 1993.

5. W. Brainerd. Tree generating regular systems. Information and Control, 14:217-231, 1969.

6. J. R. Büchi. Regular canonical systems. Archiv für Mathematische Logik und Grundlagenforschung, 6:91-111, 1964.

7. P. Cartier and D. Foata. Problèmes combinatoires de commutation et réarrangements. Number 85 in Lecture Notes in Mathematics. Springer, 1969.

8. D. Caucal. Monadic theory of term rewriting. In Proceedings of the 7th Annual IEEE Symposium on Logic in Computer Science (LICS '92), pages 266-273. IEEE Computer Society Press, 1990.

9. D. Caucal. On the regular structure of prefix rewriting. Theoretical Computer Science, 106:61-86, 1992.

10. D. Caucal. On infinite transition graphs having a decidable monadic theory. In F. M. auf der Heide and B. Monien, editors, Proceedings of the 23nd International Colloquium on Automata, Languages and Programming (ICALP'96), Paderborn (Germany), number 1099 in Lecture Notes in Computer Science, pages 194-205. Springer, 1996.

11. K. J. Compton and C. W. Henson. A uniform method for proving lower bounds on the computational complexity of logical theories. Annals of Pure and Applied Logic, 48:1-79, 1990.

12. R. Cori and Y. Métivier. Recognizable subsets of some partially abelian monoids. Theoretical Computer Science, 35:179-189, 1985.

13. R. Cori and D. Perrin. Automates et commutations partielles. R.A.I.R.O. - Informatique Théorique et Applications, 19:21-32, 1985.

14. M. Dauchet and S. Tison. The theory of ground rewrite systems is decidable. In Proceedings of the 5th Annual IEEE Symposium on Logic in Computer Science (LICS '90), pages 242-256. IEEE Computer Society Press, 1990.

15. V. Diekert. On the Knuth-Bendix completion for concurrent processes. In T. Ottmann, editor, Proceedings of the14th International Colloquium on Automata, Languages and Programming (ICALP 87), Karlsruhe (Germany), number 267 in Lecture Notes in Computer Science, pages 42-53. Springer, 1987.

16. V. Diekert. Combinatorics on Traces. Number 454 in Lecture Notes in Computer Science. Springer, 1990.

17. V. Diekert, Y. Matiyasevich, and A. Muscholl. Solving word equations modulo partial commutations. Theoretical Computer Science, 224(1-2):215-235, 1999.

18. V. Diekert and G. Rozenberg, editors. The Book of Traces. World Scientific, Singapore, 1995.

19. M. Droste and D. Kuske. Trace languages definable with modular quantifiers. In J. Dassow, G. Rozenberg, and A. Salomaa, editors, Developments in Language Theory II, At the Crossroads of Mathematics, Computer Science and Biology, Magdeburg (Germany), pages 130-144. World Scientific, 1995.

20. C. Duboc. On some equations in free partially commutative monoids. Theoretical Computer Science, 46:159174, 1986.

21. V. G. Durnev. Undecidability of the positive $\forall \exists^{3}$-theory of a free semi-group. Sibirsky Matematicheskie Jurnal, 36(5):1067-1080, 1995. English translation.

22. W. Ebinger and A. Muscholl. Logical definability on infinite traces. Theoretical Computer Science, 154(1):6784, 1996.

23. S. Eilenberg. Automata, Languages, and Machines, volume A. Academic Press, New York and London, 1974.

24. C. Frougny and J. Sakarovitch. Synchronized rational relations of finite and infinite words. Theoretical Computer Science, 108(1):45-82, 1993.

25. H. Gaifman. On local and nonlocal properties. In J. Stern, editor, Logic Colloquium '81, pages 105-135. North Holland, 1982.

26. Y. Gurevich. Monadic second-order theories. In J. Barwise and S. Feferman, editors, Model-Theoretic Logics, pages 479-506. Springer, 1985.

27. W. Hodges. Model Theory. Cambridge University Press, 1993.

28. G. Huet and D. Lankford. On the uniform halting problem for term rewriting systems. Report Lab. Report No. 283, INRIA, Le Chesnay, France, 1978.

29. F. Jacquemard. Automates d'arbres et Réécriture de termes. PhD thesis, Université de Paris-Sud, 1996. In French.

30. M. Jantzen. Confluent string rewriting. In EATCS Monographs on theoretical computer science, volume 14. Springer, 1988.

31. B. Khoussainov and A. Nerode. Automatic presentations of structures. In LCC: International Workshop on Logic and Computational Complexity, number 960 in Lecture Notes in Computer Science, pages 367-392, 1994. 
32. D. E. Knuth and P. B. Bendix. Simple Word Problems in Universal Algebras. In J. Leech, editor, Computational Problems in Abstract Algebras, pages 263-297. Pergamon Press, Elmsford N.Y., 1967.

33. D. C. Kozen. Complexity of finitely presented algebras. In 9th Annual Symposium on Theory of Computing (STOC 77), pages 164-177. ACM Press, 1977.

34. L. Libkin. On counting logics and local properties. In Proceedings of the 13th Annual IEEE Symposium on Logic in Computer Science (LICS'98), pages 501-512. IEEE Computer Society Press, 1998.

35. L. Libkin. Logics with counting and local properties. ACM Transactions on Computational Logic (TOCL), 1(1):33-59, 2000.

36. L. Libkin. Logics capturing local properties. ACM Transactions on Computational Logic (TOCL), 2(1):135$153,2001$.

37. C. Löding:. Ground tree rewriting graphs of bounded tree width. In H. Alt and A. Ferreira, editors, Proceedings of the 19th Annual Symposium on Theoretical Aspects of Computer Science (STACS 2002), Juan les Pins (France), number 2285 in Lecture Notes in Computer Science, pages 559-570. Springer, 2002.

38. M. Lohrey. On the confluence of trace rewriting systems. In V. Arvind and R. Ramanujam, editors, Proceedings of the 18th Conference on Foundations of Software Technology and Theoretical Computer Science, (FSTTCS'98), Chennai (India), number 1530 in Lecture Notes in Computer Science, pages 319-330. Springer, 1998.

39. M. Lohrey. Das Konfluenzproblem für Spurersetzungssysteme. PhD thesis, Universität Stuttgart, 1999. In German.

40. M. Lohrey. Confluence problems for trace rewriting systems. Information and Computation, 170:1-25, 2001.

41. M. Lothaire. Combinatorics on Words, volume 17 of Encyclopedia of Mathematics and its Applications. Addison-Wesley, Reading, MA, 1983.

42. S. S. Marchenkov. Unsolvability of the positive $\forall \exists$-theory of a free semi-group. Sibirsky Matematicheskie Jurnal, 23(1):196-198, 1982.

43. A. Markov. On the impossibility of certain algorithms in the theory of associative systems. Doklady Akademii Nauk SSSR, 55, 58:587-590, 353-356, 1947.

44. Y. Matiyasevich. Some decision problems for traces. In S. Adian and A. Nerode, editors, Proceedings of the 4th International Symposium on Logical Foundations of Computer Science (LFCS'97), Yaroslavl (Russia), number 1234 in Lecture Notes in Computer Science, pages 248-257. Springer, 1997.

45. O. Matz. Dot-depth, monadic quantifier alternation, and first-order closure over grids and pictures. Theoretical Computer Science, 270(1-2):1-70, 2002.

46. A. Mazurkiewicz. Concurrent program schemes and their interpretations. DAIMI Rep. PB 78, Aarhus University, Aarhus, 1977.

47. A. R. Meyer. Weak monadic second order theory of one successor is not elementary recursive. In Proceedings of the Logic Colloquium (Boston 1972-73), number 453 in Lecture Notes in Mathematics, pages 132-154. Springer, 1975.

48. C. Morvan. On rational graphs. In J. Tiuryn, editor, Proceedings of the 3rd International Conference on Foundations of Software Science and Computation Structures (FoSSaCS 2000), Berlin (Germany), number 2303 in Lecture Notes in Computer Science, pages 252-266. Springer, 2002.

49. C. Morvan and C. Stirling. Rational graphs trace context-sensitive languages. In J. Sgall, A. Pultr, and P. Kolman, editors, Proceedings of the 26th International Symposium on Mathematical Foundations of Computer Science (MFCS 2001), Marianske Lazne (Czech Republic), number 2136 in Lecture Notes in Computer Science, pages 548-559. Springer, 2001.

50. D. E. Muller and P. E. Schupp. The theory of ends, pushdown automata, and second-order logic. Theoretical Computer Science, 37(1):51-75, 1985.

51. P. Narendran and F. Otto. Preperfectness is undecidable for Thue systems containing only length-reducing rules and a single commutation rule. Information Processing Letters, 29:125-130, 1988.

52. M. H. A. Newman. On theories with a combinatorial definition of "equivalence". Annals of Mathematics, 43:223-243, 1943.

53. J. Nurmonen. Counting modulo quantifiers on finite structures. Information and Computation, 160(1-2):6287, 2000.

54. E. Post. Recursive unsolvability of a problem of Thue. Journal of Symbolic Logic, 12(1):1-11, 1947.

55. M. Presburger. Über die Vollständigkeit eines gewissen Systems der Arithmetik ganzer Zahlen, in welchem die Addition als einzige Operation hervortritt. In Comptes Rendus du Premier Congrès des Mathématicienes des Pays Slaves, pages 92-101, Warsaw, 1929.

56. W. V. O. Quine. Concatenation as a basis for arithmetic. Journal of Symbol Logic, 11(4):105-114, 1946. 
57. M. O. Rabin. Decidability of second-order theories and automata on infinite trees. Transactions of the American Mathematical Society, 141:1-35, 1969.

58. K. Reinhardt. The complexity of translating logic to finite automata. In E. Grädel, W. Thomas, and T. Wilke, editors, Automata, Logics, and Infinite Games, number 2500 in Lecture Notes in Computer Science, pages 231-238. Springer, 2002.

59. H. Straubing, D. Thérien, and W. Thomas. Regular languages defined with generalized quanifiers. Information and Computation, 118(2):289-301, 1995.

60. Terese. Term rewriting systems. Cambridge University Press, 2003.

61. W. Thomas. Automata on infinite objects. In J. van Leeuwen, editor, Handbook of Theoretical Computer Science, chapter 4, pages 133-191. Elsevier Science Publishers B. V., 1990.

62. W. Thomas. On logical definability of trace languages. In V. Diekert, editor, Proceedings of a workshop of the ESPRIT Basic Research Action No 3166: Algebraic and Syntactic Methods in Computer Science (ASMICS), Kochel am See (Germany), Report TUM-I9002, Technical University of Munich, pages 172-182, 1990.

63. A. Thue. Probleme über die Veränderungen von Zeichenreihen nach gegebenen Regeln. Skr. Vid. Kristiania, I Math. Natuv. Klasse, No. 10, 34 S., 1914. In German.

64. S. Tison. Automates comme outil de décision dans les arbres. Dossier d'habilitation à diriger des recherches, Université des Sciences et Techniques de Lille, 1990.

65. R. Treinen. The first-order theory of linear one-step rewriting is undecidable. Theoretical Computer Science, 208(1-2):149-177, 1998. 